ESAIM: PROCEEDINGS, December 2012, Vol. 38, p. 220-240

F. Coquel, M. Gutnic, P. Helluy, F. Lagoutière, C. Rohde, N. Seguin, Editors

\title{
NUMERICAL SIMULATION BY A RANDOM PARTICLE METHOD OF DEUTERIUM-TRITIUM FUSION REACTIONS IN A PLASMA*
}

\author{
Fréderique Charles, Stéphane Dellacherie ${ }^{1}$ and Jean-Marc Mounsamy ${ }^{2}$
}

\begin{abstract}
We propose and we justify a Monte-Carlo algorithm which solves a spatially homogeneous kinetic equation of Boltzmann type that models the fusion reaction between a deuterium ion and a tritium ion, and giving an $\alpha$ particle and a neutron. The proposed algorithm is validated with the use of explicit solutions of the kinetic model obtained by replacing the fusion cross-section by a Maxwellian cross section.

Résumé. On propose et on justifie un algorithme de type Monte-Carlo permettant de résoudre un modèle cinétique homogène en espace de type Boltzmann modélisant la réaction de fusion entre un ion deutérium et un ion tritium, et donnant une particule $\alpha$ et un neutron. L'algorithme proposé est par ailleurs validé via des solutions explicites du modèle cinétique obtenues en remplaçant la section efficace de fusion par une section efficace maxwellienne.
\end{abstract}

\section{INTRODUCTION}

One of the goals of the future International Thermonuclear Experimental Reactor is to obtain fusion reactions between two isotopes of hydrogen in a confined plasma. We study here one of the possible reaction: $\mathrm{T}(\mathrm{d}, \mathrm{n})^{4} \mathrm{He}$, between Deuterium $(D)$ and Tritium $(T)$ ions producing an $\alpha$ particle and a neutron $(\mathrm{n})$ with the following scheme:

$$
D+T \rightarrow \alpha+n+17,59 \mathrm{MeV} .
$$

When two particles of Deuterium and Tritium fuse, they disappear and produce an $\alpha$ particle and a neutron with a mass default $\Delta M$. According to Einstein's law, the energy produced by this mass is $Q:=\Delta M c^{2}=17,59$ $\mathrm{MeV}$ (where $c$ is the light speed). Here we assume that this energy is fully converted into kinetic energy. The very energetic $\alpha$ particles produced have a significant role in ITER: they take part in the heating of the plasma, contribute to the evolution of the electrical field and can cause instabilities. For these reasons, the study of their trajectories and their interactions with other species is an important point for the comprehension of instabilities in a fusion plasma. In [5] for example, the $\alpha$ particles current drive and heating is studied, using an expression of the fusion reaction rate $\tau$ which is the rate of $\alpha$ particles produced per unit volume per second (see $\S 1.2$ ). However, in areas where the plasma may not be at thermodynamical equilibrium, it is more appropriate - in particular because classical expressions of the nuclear fusion rate are not true - to use a kinetic modelling and to compute the velocities distribution of the $\alpha$ particles. In [3], Dellacherie and Sentis introduced a kinetic model

\footnotetext{
* The authors would like to thank the financial support of the Large Scale Initiative FUSION of INRIA.

${ }^{1}$ Laboratoire de Modélisation et simulation à l'Échelle Composant, CEA, DEN, DM2S, STMF,

F-91191 Gif-sur-Yvette Cedex, France.
}

(C) EDP Sciences, SMAI 2012 
to describe nuclear collisions. In a spatially homogeneous context, the model writes:

$$
\left\{\begin{array}{l}
\frac{\partial f_{D}}{\partial t}=-P_{D}\left(f_{D}, f_{T}\right), \quad \text { (a) } \\
\frac{\partial f_{T}}{\partial t}=-P_{T}\left(f_{D}, f_{T}\right), \quad \text { (b) } \\
\frac{\partial f_{\alpha}}{\partial t}=G_{\alpha}\left(f_{D}, f_{T}\right), \quad \text { (c) } \\
\frac{\partial f_{n}}{\partial t}=G_{n}\left(f_{D}, f_{T}\right), \quad \text { (d) }
\end{array}\right.
$$

where $f_{a}: \mathbb{R}^{+} \times \mathbb{R}^{3} \mapsto \mathbb{R}^{+}$is a non negative function, for $a \in\{D, T, \alpha, n\} ; f_{a}\left(t, v_{a}\right)$ corresponds to the number densities $^{1}$ in ions Deuterium, ions Tritium, neutrons, and $\alpha$ particles respectively at time $t \in \mathbb{R}^{+}$at velocity $v_{a} \in \mathbb{R}^{3}$. In (1), operators $P_{a}\left(f_{D}, f_{T}\right), a \in\{D, T\}$ are loss operators, which model the disappearing of a $D$ or $T$ particle after a collision, whereas $G_{a}\left(f_{D}, f_{T}\right), a \in\{\alpha, n\}$ are gain operators, corresponding to the creation of a neutron or an $\alpha$ particle. An asymptotic analysis of the gain operator when the energy of the reaction $Q \rightarrow+\infty$ is also performed, and leads to an approached model where the gain operators are substituted by Dirac masses on $\mathbb{S}^{2}$.

We propose here a random particle method simulation of the model (1), and we compare this last model with another model obtained as a limit when the ratio between the kinetic temperature of the plasma and $Q$ tends to 0 . The paper is organized as follows. In the first Section, we present the collision model introduced by Dellacherie and Sentis in [3] and the asymptotic model. We also establish expression of explicit solutions in the case of a Maxwellian cross-section of collision. In the second Section, we present the numerical method we developed to solve the model (1). This method is based on a Monte-Carlo simulation of the loss and gain operators, and is close from the Nanbu's method for Boltzmann operator. The third Section is devoted to numerical results. First, we validate our algorithm thanks to explicit solutions obtained in the case of the Maxwellian cross-section. Then, we present the numerical simulation of model (1) with the fusion cross-section. Finally, we investigate the behaviour of the function density in energy of $\alpha$ particles when the kinetic temperature of the plasma decreases, in order to discuss the opportunity of using an asymptotic model.

\section{Kinetic modeling of D-T fusion Reaction}

\subsection{Collision operators}

The model established in [3] is based on the following hypothesis:

- the energy corresponding to the mass default $Q=\Delta M c^{2}$ is totally converted into kinetic energy on $\alpha$ particles and neutron,

- we neglect the mass default $\Delta M$ with respect to the masses of particles which means that we assume that

$$
m_{D}+m_{T}=m_{\alpha}+m_{n}
$$

where $m_{D}, m_{T}, m_{\alpha}$ and $m_{n}$ are the masses of ions Deuterium, Tritium, $\alpha$ and of a neutron respectively,

- the momentum is conserved during a collision.

Consequently, the momentum conservation and the energy balances write

$$
\begin{aligned}
m_{D} v_{D}+m_{T} v_{T} & =m_{\alpha} v_{\alpha}+m_{n} v_{n} \\
m_{D} v_{D}^{2}+m_{T} v_{T}^{2}+2 Q & =m_{\alpha} v_{\alpha}^{2}+m_{n} v_{n}^{2}
\end{aligned}
$$

\footnotetext{
${ }^{1} f_{a}\left(t, v_{a}\right)$ is in $\mathrm{s}^{3} \cdot \mathrm{m}^{-6}$
} 
Let us define the following notations:

$$
\mu_{D T}=\frac{m_{D} m_{T}}{m_{D}+m_{T}}, \quad \mu_{\alpha n}=\frac{m_{\alpha} m_{n}}{m_{\alpha}+m_{n}}, \quad \zeta=\sqrt{2 Q / \mu_{\alpha n}} .
$$

From (3), we deduce that there exists $\Omega \in \mathbb{S}^{2}$ such that the velocities of the created particles $v_{\alpha}$ and $v_{n}$ write

$$
\begin{aligned}
& v_{\alpha}=v_{\alpha}\left(v_{D}, v_{T}, \Omega\right)=\frac{m_{D} v_{D}+m_{T} v_{T}}{m_{D}+m_{T}}+\Omega \frac{\mu_{\alpha n}}{m_{\alpha}} \sqrt{\zeta^{2}+\frac{\mu_{D T}}{\mu_{\alpha n}}\left|v_{D}-v_{T}\right|^{2}}, \\
& v_{n}=v_{\alpha}\left(v_{D}, v_{T}, \Omega\right)=\frac{m_{D} v_{D}+m_{T} v_{T}}{m_{D}+m_{T}}-\Omega \frac{\mu_{\alpha n}}{m_{n}} \sqrt{\zeta^{2}+\frac{\mu_{D T}}{\mu_{\alpha n}}\left|v_{D}-v_{T}\right|^{2}} .
\end{aligned}
$$

It is then possible to derive the expression of the loss and gain operators. The loss operator $P_{D}$ writes (we have a similar expression for $\left.P_{T}\right)$ :

$$
P_{D}\left(f_{D}, f_{T}\right)\left(v_{D}\right)=f_{D}\left(v_{D}\right) \int_{\mathbb{R}^{3}} \int_{\mathbb{S}^{2}} f_{T}\left(v_{T}\right) q\left(\left|v_{D}-v_{T}\right|, \Omega\right) d v_{T} d \Omega
$$

and the gain operator $G_{\alpha}$ writes on a weak form ${ }^{2}$ (we have a similar expression for $G_{n}$ ):

$$
\int_{\mathbb{R}^{3}} G_{\alpha}\left(f_{D}, f_{T}\right)(v) \varphi(v) d v=\int_{\mathbb{R}^{3}} \int_{\mathbb{R}^{3}} \int_{\mathbb{S}^{2}} f_{D}\left(v_{D}\right) f_{T}\left(v_{T}\right) \varphi\left(v_{\alpha}\right) q\left(\left|v_{D}-v_{T}\right|, \Omega\right) d v_{D} d v_{T} d \Omega,
$$

where $v_{\alpha}$ depends on $v_{D}, v_{T}$ and $\Omega$ through (5). The collision kernel $q$ is here isotropic - that is $q\left(\left|v_{D}-v_{T}\right|, \Omega\right)=$ $q\left(\left|v_{D}-v_{T}\right|\right)-$ and is defined by

$$
q\left(\left|v_{D}-v_{T}\right|\right)=\left|v_{D}-v_{T}\right| \sigma\left(\left|v_{D}-v_{T}\right|\right)
$$

where $\sigma\left(\left|v_{D}-v_{T}\right|\right)$ is called the collision cross-section. We will here consider two types of collision cross-section:

- the nuclear cross-section which has the following form ${ }^{3}$

$$
\sigma_{f}\left(\left|v_{D}-v_{T}\right|\right)=\frac{p_{5}+p_{2} /\left[\left(p_{4}-p_{3} E\right)^{2}+1\right]}{E\left[\exp \left(p_{1} / \sqrt{E}\right)-1\right]}
$$

where $E=\frac{1}{2} m_{D}\left|v_{D}-v_{T}\right|^{2}$ and $p_{i} \in \mathbb{R}^{+}$are the Duane coefficients $[4,8]$,

- the Maxwellian cross-section ${ }^{4}$ given by

$$
\sigma_{m}\left(\left|v_{D}-v_{T}\right|\right)=\frac{\lambda}{4 \pi\left|v_{D}-v_{T}\right|}
$$

where $\lambda$ is a constant (in $\left.\mathrm{m}^{3} \cdot \mathrm{s}^{-1}\right)$. Thus, we have $q\left(\left|v_{D}-v_{T}\right|\right)=\lambda / 4 \pi$. We use this cross-section to obtain explicit solutions which enable us to validate the numerical method.

Remark 1. It should be possible to prove the existence and uniqueness of non-negative solutions of (1) with initial conditions with appropriated assumptions on $q$ and on the initial condition in the same spirit as in [2]. However, we do not study this problem here and we assume that the moments of order 0,1 and 2 in velocity of the density functions $f_{a}(a \in\{D, T, \alpha, n\})$ are well defined.

\footnotetext{
${ }^{2}$ It is also possible to obtain an expression of $G_{\alpha}$ on a strong form [3] but we do not need it here.

${ }^{3}$ Other expressions of fusion cross-section could be found, such the one of [11].

${ }^{4}$ The Maxwellian cross-section, which corresponds to the cross-section of the model of "Maxwellian molecules" for Boltzmann operator, should be considered as a theoretical model. However, it is widely used in kinetic theory (see [14]) to perform many explicit calculations.
} 


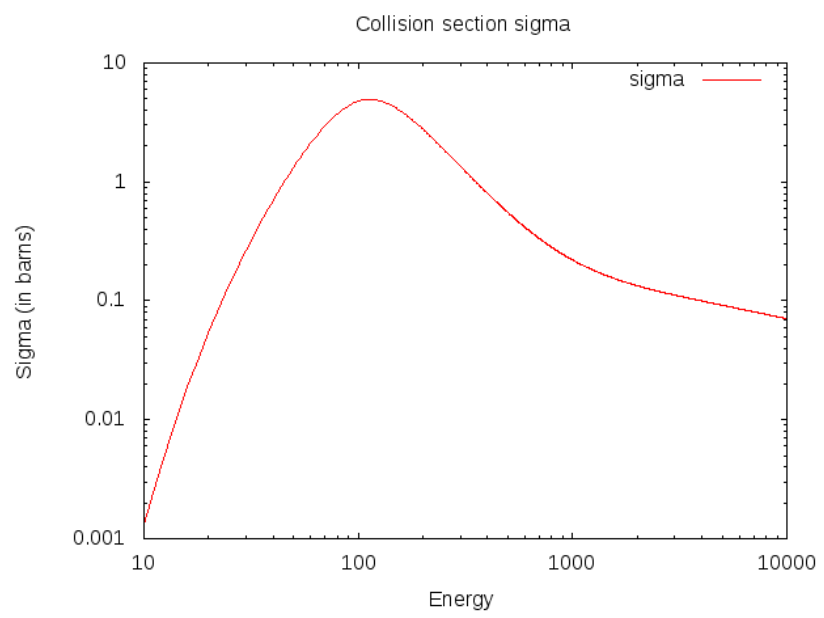

Figure 1. Cross-section $\sigma_{f}$ (in Barns, 1 Barn $=10^{-28} \mathrm{~m}^{2}$ ) according to $E$ (in $\mathrm{keV}$ ) in log-log scale.

\subsection{Definition of some physical quantities}

We define here some physical quantities used in the following. For each species $a \in\{D, T, \alpha, n\}$, we define the number density $\rho_{a}\left(\right.$ in $\left.\mathrm{m}^{-3}\right)$, the macroscopic velocity $u_{a}$ (in $\mathrm{m} \cdot \mathrm{s}^{-1}$ ) and the kinetic temperature $\theta_{a}$ (here in Joules $^{5}$ ) with

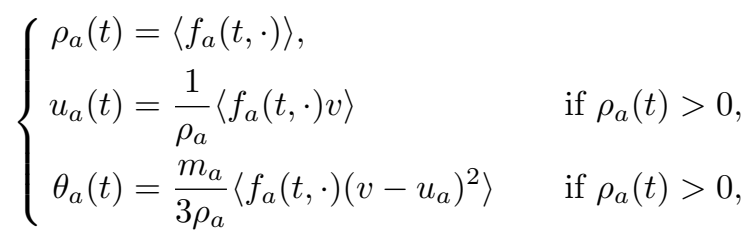

where $\langle g\rangle$ represents the average in velocity of a function $g \in \mathrm{L}_{v}^{1}$, that is $\int_{\mathbb{R}^{3}} g(v) d v$. We also define the fusion reaction rate which corresponds to the number of particles $\alpha$ (or of neutrons) created per unit of volume and of time:

$$
\tau(t)=\tau\left(f_{D}, f_{T}\right)(t):=\int_{\mathbb{R}^{3}} \int_{\mathbb{R}^{3}} \int_{\mathbb{S}^{2}} f_{D}\left(t, v_{D}\right) f_{T}\left(t, v_{T}\right) q\left(\left|v_{D}-v_{T}\right|, \Omega\right) d \Omega d v_{T} d v_{D}
$$

the total momentum $W$ :

$$
W(t)=m_{D} \rho_{D} u_{D}+m_{T} \rho_{T} u_{T}+m_{\alpha} \rho_{\alpha} u_{\alpha}+m_{n} \rho_{n} u_{n}
$$

and the total kinetic energy $E_{\text {tot }}$ :

$$
2 E_{\mathrm{tot}}(t)=m_{D}\left\langle f_{D}\left(t, v_{D}\right) v_{D}^{2}\right\rangle+m_{T}\left\langle f_{T}\left(t, v_{T}\right) v_{T}^{2}\right\rangle+m_{\alpha}\left\langle f_{\alpha}\left(t, v_{\alpha}\right) v_{\alpha}^{2}\right\rangle+m_{n}\left\langle f_{n}\left(t, v_{n}\right) v_{n}^{2}\right\rangle .
$$

\footnotetext{
${ }^{5}$ We can also define in Kelvin the temperature $T_{a}$ of the particles of type $a$ by using the relation $\theta_{a}=k_{B} T_{a}$ where $k_{B}=1.3806 \cdot 10^{-23} \mathrm{~J} \cdot \mathrm{K}^{-1}$ is the Boltzmann constant.
} 
Let us underline that $\tau$ corresponds to $\left\langle P_{a}\left(f_{D}, f_{T}\right)\right\rangle(a \in\{D, T\})$ and to $\left\langle G_{a}\left(f_{D}, f_{T}\right)\right\rangle(a \in\{\alpha, n\})$. Moreover if $f_{a}, a \in\{D, T, \alpha, n\}$ are solutions of (1) then, thanks to relation (3), we have:

$$
\left\{\begin{array}{l}
\tau=-\frac{d}{d t} \rho_{D}=-\frac{d}{d t} \rho_{T}=\frac{d}{d t} \rho_{\alpha}=\frac{d}{d t} \rho_{n} \\
\frac{d W}{d t}=0 \\
\frac{d E_{\mathrm{tot}}}{d t}=Q \tau(t) .
\end{array}\right.
$$

\subsection{An asymptotic model}

Let us introduce the thermal velocities $V_{a}^{t h}:=\sqrt{2 \theta_{a} / m_{a}}(a \in\{D, T\}), V_{p}^{t h}=\max \left(V_{D}^{t h}, V_{T}^{t h}\right)$ and

$$
\varepsilon=\frac{V_{p}^{t h}}{\zeta}
$$

where $\zeta$ is defined by (4). For $\theta_{D}=\theta_{T}=10 \mathrm{keV}^{6}$ (which corresponds to a temperature in $\mathrm{K}$ of $T_{D}=\theta_{D} / k_{B}=$ $1.16 \cdot 10^{8} \mathrm{~K}$, which is the classical order of magnitude of the temperature in a Tokamak plasma), we have $\varepsilon=0.015$. In this situation, we see in (5) that, formally, we have

$$
\left|v_{\alpha}\right| \approx \overline{V_{\alpha}}:=\frac{\mu_{\alpha n}}{m_{\alpha}} \zeta \quad \text { and } \quad\left|v_{n}\right| \approx \overline{V_{n}}:=\frac{\mu_{\alpha n}}{m_{n}} \zeta .
$$

Then, the velocities of created particles $\alpha$ and $n$ are very close to the standard creation velocities $\overline{V_{\alpha}}$ and $\overline{V_{n}}$, which correspond to the well-known values of energy

$$
\begin{cases}\overline{E_{\alpha}}=\frac{1}{2} m_{\alpha}{\overline{V_{\alpha}}}^{2}=3.52 \mathrm{MeV} \\ \overline{E_{n}}=\frac{1}{2} m_{n}{\overline{V_{n}}}^{2}=14.08 \mathrm{MeV}\end{cases}
$$

More precisely, one can prove [3] that if $q \in \mathrm{L}^{\infty}(\mathbb{R}), f_{D}$ and $f_{T}$ are in $\mathrm{L}^{1}\left(\mathbb{R}^{3} ;\left(1+|v|^{2}\right) d v\right)$, the term $\frac{G_{a}\left(f_{D}, f_{T}\right)}{\left\langle G_{a}\left(f_{D}, f_{T}\right)\right\rangle}$ $(a \in\{\alpha, n\})$ converges weakly in the sens of measures to the Dirac mass $\frac{1}{4 \pi} \delta_{a}$ on $\mathbb{S}^{2}$ defined by

$$
\int_{\mathbb{R}^{3}} \varphi(v) \delta_{a}(v) d v=\int_{\mathbb{S}^{2}} \varphi\left(\overline{V_{a}} \Omega\right) d \Omega, \quad \forall \varphi \in \mathcal{C}\left(\mathbb{R}^{3}\right) .
$$

When $\varepsilon \ll 1$ in (1), we can therefore consider an approached model by replacing the gain operator $G_{a}$ by its weak limit, which gives

$$
\left\{\begin{aligned}
\frac{\partial f_{D}}{\partial t} & =-P_{D}\left(f_{D}, f_{T}\right) \\
\frac{\partial f_{T}}{\partial t} & =-P_{T}\left(f_{D}, f_{T}\right) \\
\frac{\partial f_{\alpha}}{\partial t} & =\frac{1}{4 \pi} \tau\left(f_{D}, f_{T}\right) \delta_{\alpha} \\
\frac{\partial f_{n}}{\partial t} & =\frac{1}{4 \pi} \tau\left(f_{D}, f_{T}\right) \delta_{n}
\end{aligned}\right.
$$

where $\tau\left(f_{D}, f_{T}\right)$ is defined by $(11)$.

\footnotetext{
${ }^{6} 1 \mathrm{keV}=1.602 \cdot 10^{-16} \mathrm{~J}$
} 
Remark 2. On the contrary of model (1), density functions solutions of model (18) do not verify equations $(14)(b, c)$.

\subsection{Explicit solutions in the case of a Maxwellian cross-section}

We have the following result :

Proposition 1. We consider the system (1) with the following initial conditions

$$
f_{D}(0, v)=f_{D}^{i n i}(v) \geq 0, \quad f_{T}(0, v)=f_{T}^{i n i}(v) \geq 0, \quad f_{\alpha}(0, v)=0, \quad f_{n}(0, v)=0,
$$

where the cross-section $\sigma$ in the operators $P_{D}, P_{T}, G_{\alpha}$ and $G_{n}$ is given by the Maxwellian cross-section $\sigma_{m}(9)$. When $0<\rho_{D}(0)=\rho_{T}(0)=$ : $\rho^{\text {ini }}$ (where $\rho_{D}$ and $\rho_{T}$ are defined by $(10)$ ), $f_{D}$ and $f_{T}$ are given by

$$
\left\{\begin{aligned}
f_{D}\left(t, v_{D}\right) & =\frac{f_{D}^{i n i}\left(v_{D}\right)}{\lambda \rho^{i n i} t+1}, \\
f_{T}\left(t, v_{T}\right) & =\frac{f_{T}^{i n i}\left(v_{T}\right)}{\lambda \rho^{i n i} t+1}
\end{aligned}\right.
$$

and $f_{\alpha}$ and $f_{n}$ are given by the weak forms

$$
\begin{aligned}
\left\langle f_{\alpha}(t, \cdot) \varphi\right\rangle & =\frac{\lambda\left(\rho^{i n i}\right)^{2} t}{\lambda \rho^{i n i} t+1} R_{\alpha}\left(f_{D}^{i n i}, f_{T}^{i n i}\right)(\varphi), \\
\left\langle f_{n}(t, \cdot) \varphi\right\rangle & =\frac{\lambda\left(\rho^{i n i}\right)^{2} t}{\lambda \rho^{i n i} t+1} R_{n}\left(f_{D}^{i n i}, f_{T}^{i n i}\right)(\varphi),
\end{aligned}
$$

for all $\varphi$ such that $\left\langle f_{a} \varphi\right\rangle(a \in\{\alpha, n\})$ is well defined, $\left.R_{a}(a \in\{\alpha, n\})\right)$ being given by

$$
R_{a}\left(f_{D}^{i n i}, f_{T}^{i n i}\right)(\varphi)=\frac{1}{4 \pi\left(\rho^{i n i}\right)^{2}} \int_{\mathbb{R}^{3}} \int_{\mathbb{R}^{3}} \int_{\mathbb{S}^{2}} f_{D}^{i n i}\left(v_{D}\right) f_{T}^{i n i}\left(v_{T}\right) \varphi\left(v_{a}\right) d v_{D} d v_{T} d \Omega, \quad a \in\{\alpha, n\},
$$

where $v_{a}=v_{a}\left(v_{D}, v_{T}, \Omega\right)$ is given by (5).

We deduce from Proposition 1 that the fusion reaction rate $\tau$ is given by

$$
\tau(t)=\frac{\lambda\left(\rho^{\mathrm{ini}}\right)^{2}}{\left(\lambda \rho^{\mathrm{ini}} t+1\right)^{2}}
$$

that the number densities are given by

$$
\rho_{D}(t)=\rho_{T}(t)=\frac{\rho^{\mathrm{ini}}}{\lambda \rho^{\mathrm{ini}} t+1}, \quad \rho_{\alpha}(t)=\rho_{n}(t)=\frac{\lambda\left(\rho^{\mathrm{ini}}\right)^{2} t}{\lambda \rho^{\text {ini }} t+1} \quad \text { for } t \geq 0,
$$

that the macroscopic velocities are given by

$$
u_{D}(t)=u_{D}(0), \quad u_{T}(t)=u_{T}(0), \quad u_{a}(t)=R_{a}\left(f_{D}^{\text {ini }}, f_{T}^{\text {ini }}\right)(v \mapsto v) \text { for } t>0, a \in\{\alpha, n\},
$$

and that the kinetic temperatures are given by

$$
\theta_{D}(t)=\theta_{D}(0), \quad \theta_{T}(t)=\theta_{T}(0), \quad \theta_{a}(t)=\frac{m_{a}}{3} R_{a}\left(f_{D}^{\mathrm{ini}}, f_{T}^{\mathrm{ini}}\right)\left(v \mapsto\left(v-u_{a}\right)^{2}\right) \text { for } t>0, a \in\{\alpha, n\}
$$


Let us remark that the time $\left(\lambda \rho^{\text {ini }}\right)^{-1}$ defines an order of magnitude of the scale of time of the fusion reaction. Moreover, we have $\lim _{t \rightarrow+\infty} \rho_{D}(t)=\lim _{t \rightarrow+\infty} \rho_{D}(t)=0$ and $\lim _{t \rightarrow+\infty} \rho_{\alpha}(t)=\lim _{t \rightarrow+\infty} \rho_{n}(t)=\rho^{\text {ini }}$.

We can also obtain explicit solutions in the case of model (18). Indeed, we have :

Proposition 2. We consider system (18) with initial conditions (19) where the cross-section $\sigma$ in the operators $P_{D}, P_{T}$ is given by the Maxwellian cross-section $\sigma_{m}(9)$. When $0<\rho_{D}(0)=\rho_{T}(0)=$ : $\rho^{\text {ini }}$ (where $\rho_{D}$ and $\rho_{T}$ are defined by (10)), $f_{\alpha}$ and $f_{n}$ are given by

$$
f_{a}\left(t, v_{a}\right)=\frac{\lambda\left(\rho^{i n i}\right)^{2} t}{\lambda \rho^{i n i} t+1} \cdot \frac{\delta_{a}\left(v_{a}\right)}{4 \pi}, \quad a \in\{\alpha, n\}
$$

and the macroscopic quantities are given by:

$$
\begin{gathered}
\rho_{\alpha}(t)=\rho_{n}(t)=\frac{\lambda\left(\rho^{i n i}\right)^{2} t}{\lambda \rho^{i n i} t+1} \quad \text { for } t \geq 0, \\
u_{\alpha}(t)=u_{n}(t)=0, \quad \theta_{\alpha}(t)=\frac{m_{\alpha}}{3}{\overline{V_{\alpha}}}^{2}, \quad \theta_{n}(t)=\frac{m_{n}}{3}{\overline{V_{n}}}^{2} \quad \text { for } t>0 .
\end{gathered}
$$

\section{Numerical Method}

The numerical resolution of systems (1) and (18) is based on a particle method coupled to a Monte-Carlo simulation of fusion operators, like in Nanbu's method $[9,12,13]$. The initial densities $f_{D}^{i n i}$ and $f_{T}^{i n i}$ are approached by the linear combination of Dirac masses

$$
f_{a}^{\mathrm{ini}}(v) \approx f_{a}^{0}(v):=\sum_{k=1}^{N_{a}^{0}} \omega_{a, k} \delta\left(v-V_{a, k}^{0}\right) \quad \text { for } a \in\{D, T\}
$$

which corresponds to a representation the distribution of particles of species $a$ by $N_{a}^{0}$ numerical particles having a numerical weight $\omega_{a, k}$ and a velocity $V_{a, k}^{0}$. In order to use a symmetric method, we choose the same numerical weight $\omega_{a, k}$ for all numerical particles and for both species $D$ and $T$. The number $N_{D}^{0}$ being fixed, we then take

$$
\omega=\frac{\rho_{D}^{\mathrm{ini}}}{N_{D}^{0}} \quad \text { and } \quad N_{T}^{0}=\frac{\rho_{T}^{\mathrm{ini}}}{\omega}
$$

The velocities $V_{a, k}^{0}$ of numerical particles are computed thanks to a Monte-Carlo method following the initial density of probability $f_{a}^{\text {ini }} / \rho_{a}^{\text {ini }}$. The derivative in time is approached by an explicit Euler scheme, and the computation of gain and loss operators is based on a Monte-Carlo simulation. Moreover, the initial densities $f_{\alpha}^{i n i}$ and $f_{n}^{i n i}$ are supposed to be equal to zero. Finally, the densities $f_{a}^{n}(a \in\{D, T, \alpha, n\})$ at any time $t^{n}$ are given by

$$
f_{a}^{n}(v)=\omega \sum_{i=1}^{N_{a}^{n}} \delta\left(v-V_{a, i}^{n}\right) \quad \text { for } a \in\{D, T, \alpha, n\}
$$

where $N_{a}^{n}$ and $\left(V_{a, i}^{n}\right)_{\left\{1 \leq i \leq N_{a}^{n}\right\}}$ are deduced from the Monte-Carlo simulation. In the sequel, we detail the MonteCarlo algorithm, firstly, for any cross-section such that $q \in \mathrm{L}^{\infty}\left(\mathbb{R}^{+}\right)$(the fusion cross-section given by (8) - see also Figure 1 - satisfies $q \in \mathrm{L}^{\infty}\left(\mathbb{R}^{+}\right)$) and, secondly, for a Maxwellian cross-section (9). We only present the algorithm for the densities $f_{D}$ and $f_{\alpha}$, the principle being the same for $f_{T}$ and $f_{n}$. 


\subsection{Fusion cross-section}

2.1.1. Other expressions of $P_{D}\left(f_{D}, f_{T}\right)$ and $G_{\alpha}\left(f_{D}, f_{T}\right)$

The computation of the terms $P_{a}\left(f_{D}^{n}, f_{T}^{n}\right)(v)$ and $G_{a}\left(f_{D}^{n}, f_{T}^{n}\right)(v)$ with a fusion cross-section is based on the following propositions (see Annex A for the proofs):

Proposition 3. Let $\varphi$ be a test function (for example $\varphi \in \mathcal{C}_{c}\left(\mathbb{R}^{3}\right)$ ) and let $P_{D}$ be the loss operator given by (6) where the collision kernel $q$ is in $L^{\infty}\left(\mathbb{R}^{+}\right)$. Let $q_{\max }$ be an upper bound of $q$. We set

$$
f_{a}^{n}(v)=\omega \sum_{i=1}^{N_{a}^{n}} \delta\left(v-V_{a, i}^{n}\right) \quad \text { for } a \in\{D, T\}
$$

We assume that

$$
\Delta t:=t^{n+1}-t^{n} \leq \frac{1}{\lambda_{\max } \omega} \min \left(\frac{1}{N_{T}^{n}}, \frac{1}{N_{D}^{n}}\right) \quad \text { with } \quad \lambda_{\max }=4 \pi q_{\max }
$$

Then, we have

$$
\Delta t \int_{\mathbb{R}^{3}} \varphi(v) P_{D}\left(f_{D}^{n}, f_{T}^{n}\right)(v) d v=\eta^{n} \frac{\omega}{\sharp P} \sum_{P} \sum_{k=1}^{\mathcal{N}^{n}} \varphi\left(V_{D, r_{k}}^{n}\right) \int_{0}^{1} \mathbb{1}_{\left\{0 \leq u \leq p_{r_{k}, s_{k}}^{n}\right\}} d u
$$

where

$$
\left\{\begin{array}{l}
\mathcal{N}^{n}=\left\lfloor N_{D}^{n} N_{T}^{n} \omega \Delta t \lambda_{\max }\right\rfloor, \\
\eta^{n}=\frac{N_{D}^{n} N_{T}^{n} \omega \Delta t \lambda_{\max }}{\mathcal{N}^{n}}, \\
p_{r_{k}, s_{k}}^{n}=\frac{q\left(\left|V_{D, r_{k}}^{n}-V_{T, s_{k}}^{n}\right|\right)}{q_{\max }},
\end{array}\right.
$$

and $P$ is the set of possible ways to make $\mathcal{N}^{n}$ distinct pairs $\left(r_{k}, s_{k}\right)_{\left\{1 \leq k \leq \mathcal{N}^{n}\right\}}$ among $\left\{1, \ldots, N_{D}^{n}\right\} \times\left\{1, \ldots, N_{T}^{n}\right\}$.

Proposition 4. Let $G_{\alpha}$ be the gain operator defined by (7). Under assumptions of Proposition 3, we have

$$
\Delta t \int_{\mathbb{R}^{3}} \varphi(v) G_{\alpha}\left(f_{D}^{n}, f_{T}^{n}\right)(v) d v=\eta^{n} \frac{\omega}{\sharp P} \sum_{P} \sum_{k=1}^{\mathcal{N}^{n}} \int_{\mathbb{S}^{2}} \int_{0}^{1} \varphi\left(V_{\alpha, r_{k}, s_{k}, \Omega}^{n}\right) \mathbb{1}_{\left\{0 \leq u \leq p_{r_{k}, s_{k}}^{n}\right\}} d u \frac{d \Omega}{4 \pi}
$$

where $V_{\alpha, r_{k}, s_{k}, \Omega}^{n}=v_{\alpha}\left(V_{D, r_{k}}^{n}, V_{T, s_{k}}^{n}, \Omega\right)$ is given by formula $(5)(a)$.

Let us note that, typically, $\mathcal{N}^{n}$ is of the order of $10^{3}$. Thus, we can approximate $\eta^{n}$ with 1.

\subsubsection{Monte-Carlo approximation}

The terms that we have obtained are then computed by a Monte-Carlo procedure. More precisely, we approach

$$
\frac{\omega}{\sharp P} \sum_{P} \sum_{k=1}^{\mathcal{N}^{n}} \varphi\left(V_{D, r_{k}}^{n}\right) \int_{0}^{1} \mathbb{1}_{\left\{0 \leq u \leq p_{r_{k}, s_{k}}^{n}\right\}} d u \quad \text { by } \quad \omega \sum_{k=1}^{\mathcal{N}^{n}} \varphi\left(V_{D, \mathbf{r}_{\mathbf{k}}}^{n}\right) \mathbb{1}_{\left\{0 \leq \mathbf{u}_{\mathbf{k}} \leq p_{\mathbf{r}_{\mathbf{k}}, \mathbf{s}_{\mathbf{k}}}^{n}\right\}}
$$

and

$$
\frac{\omega}{\sharp P} \sum_{P} \sum_{k=1}^{\mathcal{N}^{n}} \int_{\mathbb{S}^{2}} \int_{0}^{1} \varphi\left(V_{\alpha, r_{k}, s_{k}, \Omega}^{n}\right) \mathbb{1}_{\left\{0 \leq u \leq p_{r_{k}, s_{k}}^{n}\right\}} d u \frac{d \Omega}{4 \pi} \quad \text { by } \quad \omega \sum_{k=1}^{\mathcal{N}^{n}} \varphi\left(V_{\alpha, \mathbf{r}_{\mathbf{k}}, \mathbf{s}_{\mathbf{k}}, \Omega_{\mathbf{k}}}^{n}\right) \mathbb{1}_{\left\{0 \leq \mathbf{u}_{\mathbf{k}} \leq p_{\mathbf{r}_{\mathbf{k}}, \mathbf{s}_{\mathbf{k}}}^{n}\right\}}
$$

where the set of pairs $\left(\mathbf{r}_{\mathbf{k}}, \mathbf{s}_{\mathbf{k}}\right)_{\left\{1 \leq k \leq \mathcal{N}^{n}\right\}}$ is chosen randomly among $P$ with an uniform law, $\mathbf{u}_{\mathbf{k}}$ is randomly computed with an uniform law on $[0,1]$ and $\boldsymbol{\Omega}_{\mathbf{k}}$ is randomly computed with an uniform law on $\mathbb{S}^{2}$. Let us denote 
$\widetilde{P_{D}}$ and $\widetilde{G_{\alpha}}$ the approximated operators $P_{D}$ and $G_{\alpha}$ that we obtain by using respectively approximations $(37)$ and (38), which means that (by approximating $\eta^{n}$ with 1 )

$$
\left\{\begin{array}{l}
\Delta t \int_{\mathbb{R}^{3}} \varphi(v) \widetilde{P_{D}}\left(f_{D}^{n}, f_{T}^{n}\right)(v) d v=\omega \sum_{k=1}^{\mathcal{N}^{n}} \varphi\left(V_{D, \mathbf{r}_{\mathbf{k}}}^{n}\right) \mathbb{1}_{\left\{0 \leq \mathbf{u}_{\mathbf{k}} \leq p_{\mathbf{r}_{\mathbf{k}}, \mathbf{s}_{\mathbf{k}}}^{n}\right\}}, \\
\Delta t \int_{\mathbb{R}^{3}} \varphi(v) \widetilde{G_{\alpha}}\left(f_{D}^{n}, f_{T}^{n}\right)(v) d v=\omega \sum_{k=1}^{\mathcal{N}^{n}} \varphi\left(V_{\alpha, \mathbf{r}_{\mathbf{k}}, \mathbf{s}_{\mathbf{k}}, \boldsymbol{\Omega}_{\mathbf{k}}}^{n}\right) \mathbb{1}_{\left\{0 \leq \mathbf{u}_{\mathbf{k}} \leq p_{\mathbf{r}_{\mathbf{k}}, \mathbf{s}_{\mathbf{k}}}^{n}\right\}} .
\end{array}\right.
$$

Moreover, we use an explicit Euler scheme for the time discretization of equations (1). Then, if $f_{D}^{n}$ and $f_{\alpha}^{n}$ are the approximation at time $t^{n}$ of the exact densities $f_{D}\left(t^{n}, \cdot\right)$ and $f_{\alpha}\left(t^{n}, \cdot\right)$, we define $f_{D}^{n+1}$ and $f_{\alpha}^{n+1}$ by

$$
\begin{cases}f_{D}^{n+1}=f_{D}^{n}-\Delta t \widetilde{P_{D}}\left(f_{D}^{n}, f_{T}^{n}\right), & \text { (a) } \\ f_{\alpha}^{n+1}=f_{\alpha}^{n}+\Delta t \widetilde{G_{\alpha}}\left(f_{D}^{n}, f_{T}^{n}\right) & \text { (b) }\end{cases}
$$

Thus, by using (32), (39)(a) and (40)(a), we obtain

$$
\begin{aligned}
\int_{\mathbb{R}^{3}} \varphi(v) f_{D}^{n+1}(v) d v & =\omega \sum_{i=1}^{N_{D}^{n}} \varphi\left(V_{D, i}^{n}\right)-\omega \sum_{k=1}^{\mathcal{N}^{n}} \varphi\left(V_{D, \mathbf{r}_{\mathbf{k}}}^{n}\right) \mathbb{1}_{\left\{0 \leq \mathbf{u}_{\mathbf{k}} \leq p_{\mathbf{r}_{\mathbf{k}}, \mathbf{s}_{\mathbf{k}}}^{n}\right\}} \\
& =\omega \sum_{i=1}^{N_{D}^{n}} \varphi\left(V_{D, i}^{n}\right)-\omega \sum_{k \in A} \varphi\left(V_{D, \mathbf{r}_{\mathbf{k}}}^{n}\right) \\
& =\omega \sum_{k \in\left\{1, \ldots, N_{D}^{n}\right\} \backslash A} \varphi\left(V_{D, \mathbf{r}_{\mathbf{k}}}^{n}\right)
\end{aligned}
$$

where we set

$$
A=\left\{k \in\left\{1, \ldots, \mathcal{N}^{n}\right\} / 0 \leq \mathbf{u}_{\mathbf{k}} \leq p_{\mathbf{r}_{\mathbf{k}}, \mathbf{s}_{\mathbf{k}}}^{n}\right\}
$$

Thus, we have $\int_{\mathbb{R}^{3}} \varphi(v) f_{D}^{n+1}(v) d v=\omega \sum_{i=1}^{N_{D}^{n+1}} \varphi\left(V_{D, i}^{n+1}\right)$, with $N_{D}^{n+1}:=\sharp\left(\left\{1, \ldots, N_{D}^{n}\right\} \backslash A\right)$, that is to say

$$
f_{D}^{n+1}(v)=\omega \sum_{i=1}^{N_{a}^{n+1}} \delta\left(v-V_{a, i}^{n+1}\right)
$$

where $\left\{V_{D, 1}^{n+1}, \ldots, V_{D, N_{D}^{n+1}}^{n+1}\right\}$ is the set of velocities obtained when we remove $\left\{V_{D, \mathbf{r}_{1}}^{n}, \ldots, V_{D, \mathbf{r}_{\mathcal{N}^{\mathbf{n}}}}^{n}\right\}$ from $\left\{V_{D, 1}^{n}, \ldots, V_{D, N_{D}^{n}}^{n}\right\}$. In the same way, we deduce from (32), (39)(b) and (40)(b) that

$$
\begin{aligned}
\int_{\mathbb{R}^{3}} \varphi(v) f_{\alpha}^{n+1}(v) d v & =\omega \sum_{i=1}^{N_{\alpha}^{n}} \varphi\left(V_{\alpha}^{n}\right)+\omega \sum_{k=1}^{\mathcal{N}^{n}} \varphi\left(V_{\alpha, \mathbf{r}_{\mathbf{k}}, \mathbf{s}_{\mathbf{k}}, \boldsymbol{\Omega}_{\mathbf{k}}}^{n}\right) \mathbb{1}_{\left\{0 \leq \mathbf{u}_{\mathbf{k}} \leq p_{\mathbf{r}_{\mathbf{k}}, \mathbf{s}_{\mathbf{k}}}^{n}\right\}} \\
& =\omega \sum_{i=1}^{N_{\alpha}^{n+1}} \varphi\left(V_{\alpha, i}^{n+1}\right)
\end{aligned}
$$


that is to say

$$
f_{\alpha}^{n+1}(v)=\omega \sum_{k=1}^{N_{\alpha}^{n+1}} \delta\left(v-V_{\alpha, k}^{n+1}\right)
$$

where

$$
N_{\alpha}^{n+1}=N_{\alpha}^{n}+\sharp A
$$

and

$$
\left\{\begin{array}{l}
V_{\alpha, i}^{n+1}=V_{\alpha, i}^{n} \quad \text { if } \quad 1 \leq i \leq N_{\alpha}^{n} \\
\left\{V_{\alpha, i}^{n+1}, N_{\alpha}^{n}<i \leq N_{\alpha}^{n+1}\right\}=\left\{V_{\alpha, \mathbf{r}_{\mathbf{k}}, \mathbf{s}_{\mathbf{k}}, \boldsymbol{\Omega}_{\mathbf{k}}}^{n}, k \in A\right\}
\end{array}\right.
$$

\subsubsection{Algorithm}

We therefore deduce the following algorithm:

\section{Algorithm 1. Random Particle method for the model (1) with a fusion cross-section.}

At time step $t^{n}$, we perform the following operations:

i) We compute $\mathcal{N}^{n}=\left\lfloor N_{D}^{n} N_{T}^{n} \omega \Delta t \lambda_{\max }\right\rfloor$. The time step $\Delta t=t^{n+1}-t^{n}$ (which could depend of $n$ ) is such that condition (33) is satisfied. Thus, $\mathcal{N}^{n}$ is smaller than $N_{D}^{n}$ and $N_{T}^{n}$. If $\mathcal{N}^{n} \ll \min \left(N_{D}^{n}, N_{T}^{n}\right)$, one can increase $\Delta t$.

ii) We select successively $\mathcal{N}^{n}$ distinct numerical particles of $D$ (respectively of $T$ ), the $k^{\text {th }}$ particle being randomly chosen with an uniform discrete law among the $N_{D}^{n}-k+1$ (respectively $N_{T}^{n}-k+1$ ) remaining particles. This corresponds to draw the pairs $\left(\mathbf{r}_{\mathbf{k}}, \mathbf{s}_{\mathbf{k}}\right)_{k \in\left\{1, \ldots, \mathcal{N}^{n}\right\}}$ with an uniform law among the $\sharp P$ possible set of $\mathcal{N}^{n}$ pairs.

iii) For each of the $\mathcal{N}^{n}$ pairs of velocities $\left(V_{D, \mathbf{r}_{\mathbf{k}}}, V_{T, \mathbf{s}_{\mathbf{k}}}\right)$, we draw $\mathbf{u}_{\mathbf{k}}$ following an uniform law on $[0,1]$ and we compute the term

$$
p_{\mathbf{r}_{\mathbf{k}}, \mathbf{s}_{\mathbf{k}}}=\frac{q\left(V_{D, \mathbf{r}_{\mathbf{k}}}-V_{T, \mathbf{s}_{\mathbf{k}}}\right)}{q_{\max }}
$$

where $q(\xi)=|\xi| \sigma_{f}(|\xi|), \sigma_{f}$ being given by (8). If $p_{\mathbf{r}_{\mathbf{k}}, \mathbf{s}_{\mathbf{k}}}>\mathbf{u}_{\mathbf{k}}$, the two particles of velocities $V_{D, \mathbf{r}_{\mathbf{k}}}$ and $V_{T, \mathbf{s}_{\mathbf{k}}}$ interact (in other word, we have $k \in A$, where $A$ is defined by (41)).

iv) For each pair of interacting particles (that is to say for each $k \in A$ ), we draw ${ }^{7} \boldsymbol{\Omega}_{\mathbf{k}}$ with an uniform law on $\mathbb{S}^{2}$, and $V_{\alpha, \mathbf{r}_{\mathbf{k}}, \mathbf{s}_{\mathbf{k}}, \boldsymbol{\Omega}_{\mathbf{k}}}$ and $V_{n, \mathbf{r}_{\mathbf{k}}, \mathbf{s}_{\mathbf{k}}, \boldsymbol{\Omega}_{\mathbf{k}}}$ are computed thanks to formulae (5).

v) The $\sharp A$ pairs of interacting numerical particles of $D$ and $T$ are removed, and $\sharp A$ pairs of particles of $\alpha$

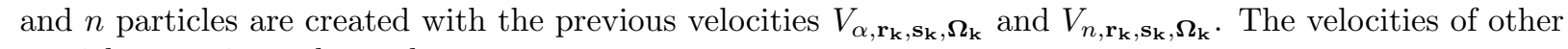
particles remain unchanged.

\footnotetext{
${ }^{7}$ We can set $\boldsymbol{\Omega}_{\mathbf{k}}=\left(a_{k} \cos \left(2 \pi \epsilon_{\mathbf{k}}\right), a_{k} \sin \left(2 \pi \epsilon_{\mathbf{k}}\right), b_{k}\right)$ with $b_{k}=1-2 \mathbf{u}_{\mathbf{k}}, a_{k}=\sqrt{1-b_{k}^{2}}, \mathbf{u}_{\mathbf{k}}$ and $\epsilon_{\mathbf{k}}$ being computed following an uniform law on $[0,1]$.
} 
Remark 3. Since a numerical fusion reaction represents $\omega$ real fusion reactions, the total number of fusion

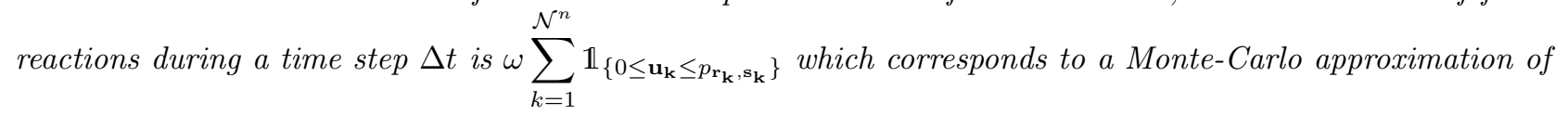

$$
\begin{aligned}
\frac{\omega}{\sharp P} \sum_{P} \sum_{k=1}^{\mathcal{N}^{n}} \int_{0}^{1} \mathbb{1}_{\left\{0 \leq u \leq p_{r_{k}, s_{k}}\right\}} d u & =\frac{\mathcal{N}^{n}}{N_{D}^{n} N_{T}^{n}} \sum_{i=1}^{N_{D}^{n}} \sum_{i=1}^{N_{T}^{n}} \omega \frac{q\left(V_{D, i}^{n}, V_{T, j}^{n}\right)}{q_{\max }} \\
& \left.\simeq \frac{\eta^{n} \mathcal{N}^{n}}{N_{D}^{n} N_{T}^{n}} \sum_{i=1}^{N_{D}^{n}} \sum_{i=1}^{N_{T}^{n}} \omega \frac{q\left(V_{D, i}^{n}, V_{T, j}^{n}\right)}{q_{\max }} \quad \quad \quad \text { since } \eta^{n} \simeq 1\right) \\
& =4 \pi \Delta t \int_{\mathbb{R}^{3}} \int_{\mathbb{R}^{3}} f_{D}^{n}\left(v_{D}\right) f_{T}^{n}\left(v_{T}\right) q\left(\left[v_{D}-v_{T} \mid\right) d v_{D} d v_{T}\right. \\
& =\Delta t \tau\left(f_{D}^{n}, f_{T}^{n}\right) \simeq \Delta t \tau\left(t^{n}\right),
\end{aligned}
$$

where $\tau\left(t^{n}\right)$ is the exact fusion reaction rate given by (11) at time $t^{n}$.

Remark 4. Algorithm 1 preserves exactly the total number of particles and the total momentum W. Moreover, the total energy verifies $E_{\text {tot }}^{n+1}=E_{\text {tot }}^{n}+Q \Delta t \tau\left(f_{D}^{n}, f_{T}^{n}\right)$. In other words, (14) is satisfied at the discrete level.

\subsection{Maxwellian cross-section}

The numerical method introduced in $\S 2.1$ for any cross-section can be simplified for a Maxwellian crosssection. Indeed, by taking $q_{\max }=q=\frac{\lambda}{4 \pi}$, we have $p_{i, j}=1$ for all couple of velocity $\left(V_{D, i}, V_{T, j}\right)$, and then

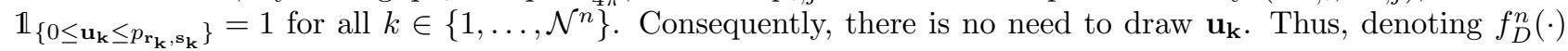
and $f_{\alpha}^{n}(\cdot)$ the approximations of the exact densities $f_{D}\left(t^{n}, \cdot\right)$ and $f_{\alpha}\left(t^{n}, \cdot\right)$ at time $t^{n}$, we approach $f_{D}\left(t^{n+1}, \cdot\right)$ and $f_{\alpha}\left(t^{n+1}, \cdot\right)$ with

$$
f_{D}^{n+1}(v)=\omega \sum_{k=1}^{N_{D}^{n+1}} \delta\left(v-V_{D, k}^{n+1}\right) \quad \text { and } \quad f_{\alpha}^{n+1}(v)=\omega \sum_{k=1}^{N_{\alpha}^{n+1}} \delta\left(v-V_{\alpha, k}^{n+1}\right)
$$

where $\left\{V_{D, 1}^{n+1}, \ldots, V_{D, N_{D}^{n+1}}^{n+1}\right\}$ is the set of velocities obtained when we remove $\left\{V_{D, \mathbf{r}_{1}}^{n}, \ldots, V_{D, \mathbf{r}_{\mathcal{N}^{\mathbf{n}}}}^{n}\right\}$ from $\left\{V_{D, 1}^{n}, \ldots, V_{D, N_{D}^{n}}^{n}\right\}$, $N_{\alpha}^{n+1}=N_{\alpha}^{n}+\mathcal{N}^{n}$ and

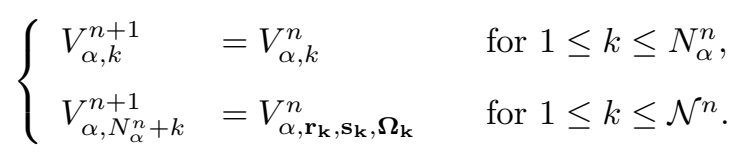

This leads to the following simplified algorithm:

\section{Algorithm 2. Random Particle method for the model (1) with Maxwellian cross-section:}

At time step $t^{n}$, the steps (i) and (ii) are the same as in Algorithm 1. Steps (iii)-(v) are replaced by:

iv) For each of the $\mathcal{N}^{n}$ pairs of velocities $\left(V_{D, \mathbf{r}_{\mathbf{k}}}, V_{T, \mathbf{s}_{\mathbf{k}}}\right)$ selected, we compute $\boldsymbol{\Omega}_{\mathbf{k}}$ with an uniform law on $\mathbb{S}^{2}$, and $V_{\alpha, \mathbf{r}_{\mathbf{k}}, \mathbf{s}_{\mathbf{k}}, \boldsymbol{\Omega}_{\mathbf{k}}}$ and $V_{n, \mathbf{r}_{\mathbf{k}}, \mathbf{s}_{\mathbf{k}}, \boldsymbol{\Omega}_{\mathbf{k}}}$ are computed thanks to formulae (5).

v) The $\mathcal{N}^{n}$ selected pairs of numerical particles of $D$ and $T$ are removed, and $\mathcal{N}^{n}$ pairs of $\alpha$ and $n$ particles

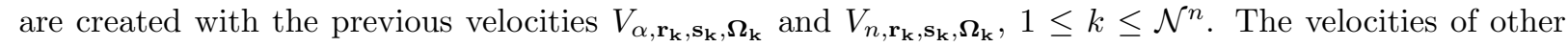
particles remain the same. 


\subsection{Asymptotic model}

We now consider the system (18) with a collision cross-section $q \in \mathrm{L}^{\infty}$ in the operators $P_{a}\left(f_{D}, d_{T}\right)(a \in$ $\{D, T\})$. This system is an approximation of system (1). The numerical resolution of equations $(18)(\mathrm{a}, \mathrm{b})$ does not change since $(18)(\mathrm{a}, \mathrm{b})$ and $(1)(\mathrm{a}, \mathrm{b})$ are identical. For the resolution of $(18)(\mathrm{c}, \mathrm{d})$, we write (thanks to definition (17) of $\left.\delta_{a}\right)$ the equation on $f_{a}(a \in\{\alpha, n\})$ on the following weak form:

$$
\frac{d}{d t}\left\langle f_{a}(t, \cdot) \varphi\right\rangle=\tau\left(f_{D}, f_{T}\right)(t) \int_{\mathbb{S}^{2}} \varphi\left(\overline{V_{a}} \Omega\right) \frac{d \Omega}{4 \pi} .
$$

Similar computations to those of Proposition 4 show that under condition (33) on the time step $\Delta t$, we can approach by a Monte-Carlo procedure

$$
\Delta t \tau\left(f_{D}^{n}, f_{T}^{n}\right) \int_{\mathbb{S}^{2}} \varphi\left(\overline{V_{a}} \Omega\right) \frac{d \Omega}{4 \pi} \quad \text { by } \quad \eta^{n} \omega \sum_{k=1}^{\mathcal{N}^{n}} \varphi\left(\overline{V_{a}} \boldsymbol{\Omega}_{\mathbf{k}}\right) \mathbb{1}_{\left\{0 \leq \mathbf{u}_{\mathbf{k}} \leq p_{\mathbf{r}_{\mathbf{k}}, \mathbf{s}_{\mathbf{k}}}\right\}}
$$

where $\mathbf{u}_{\mathbf{k}}, \mathbf{r}_{\mathbf{k}}, \mathbf{s}_{\mathbf{k}}$ are the random variables drawn for the numerical simulation of equations (1)(a,b) (steps (ii) and (iii) of Algorithm 1), and where $\left(\boldsymbol{\Omega}_{\mathbf{k}}\right)_{k \in\left\{1, \ldots, \mathcal{N}^{n}\right\}}$ are drawn following an uniform law on $\mathbb{S}^{2}$. Thus, by applying as previously an Euler scheme for the time discretization, we obtain (by using the fact that $\eta^{n} \simeq 1$ )

$$
\left\langle f_{a}^{n+1} \varphi\right\rangle=\left\langle f_{a}^{n} \varphi\right\rangle+\omega \sum_{k=1}^{\mathcal{N}^{n}} \varphi\left(\overline{V_{a}} \boldsymbol{\Omega}_{\mathbf{k}}\right) \mathbb{1}_{\left\{0 \leq \mathbf{u}_{\mathbf{k}} \leq p_{\mathbf{r}_{\mathbf{k}}, \mathbf{s}_{\mathbf{k}}}\right\}}
$$

Finally, we obtain

$$
f_{a}^{n+1}(v)=\omega \sum_{k=1}^{N_{a}^{n+1}} \delta\left(v-V_{a, k}^{n+1}\right) \quad \text { for } a \in\{\alpha, n\}
$$

with $N_{a}^{n+1}=N_{a}^{n}+\sharp A$ and

$$
\left\{\begin{array}{l}
V_{a, i}^{n+1}=V_{a, i}^{n} \quad \text { for } \quad 1 \leq i \leq N_{a}^{n}, \\
\left\{V_{a, i}^{n+1}, N_{a}^{n}<i \leq N_{a}^{n+1}\right\}=\left\{\overline{V_{a}} \boldsymbol{\Omega}_{\mathbf{k}}, k \in A\right\},
\end{array}\right.
$$

$A$ being defined by (41). This leads to the following simplified algorithm:

\section{Algorithm 3. Random Particle method for the model (18) with fusion cross-section.}

At time step $t^{n}$, steps (i) and (iii) are the same as in Algorithm 1. Steps (iv)-(v) are replaced by:

iv) The $\sharp A$ pairs of interacting numerical particles of $D$ and $T$ are removed. The velocities of other particles of species $D$ and $T$ remain the same.

v) We draw $\sharp A$ vectors of $\mathbb{S}^{2} \boldsymbol{\Omega}_{1}, \ldots \boldsymbol{\Omega}_{\sharp \mathrm{A}}$ uniformly on $\mathbb{S}^{2}$, and $\sharp A$ pairs of particles of $\alpha$ and $n$ are created with velocities $\left\{\overline{V_{a}} \boldsymbol{\Omega}_{\mathbf{k}}, k \in A\right\}$. The velocities of other particles $\alpha$ and $n$ remain the same.

Remark 5. We can easily adapt Algorithm 3 for a Maxwellian cross-section: the step (iii) is removed, and we replace $\sharp A$ by $\mathcal{N}^{n}$ in steps (iv) and (v).

Remark 6. We see that the model (18) is as costly to compute as model (1), and therefore does not present any numerical interest. However, if we are only interested in the evolution of $f_{\alpha}$ and $f_{n}$, we can introduce the 
following model

$$
\left\{\begin{aligned}
\frac{\partial f_{\alpha}}{\partial t} & =\frac{1}{4 \pi} \tau(t) \delta_{\alpha} \\
\frac{\partial f_{n}}{\partial t} & =\frac{1}{4 \pi} \tau(t) \delta_{n}
\end{aligned}\right.
$$

where $\tau(t)$ is a given fusion reaction rate. Then, at each time step, we only need to compute the step (v) of Algorithm 3 with $\left\lfloor\frac{\Delta t \tau\left(t^{n}\right)}{\omega}\right\rfloor$ instead of $\sharp A$, w being the numerical weight we choose for numerical particles. The numerical cost of this algorithm is lower than the one of Algorithms 1, 2 and 3.

\section{NumericAl RESUlts}

We now present results obtained with a code (3 dimensional in velocity) developed following methods proposed in Section 2 to solve model (1) with initial condition (19). The initial density functions $f_{D}^{\text {ini }}(v)$ and $f_{T}^{\text {ini }}(v)$ are Maxwellian distributions of density $\rho_{a}^{\text {ini }}$, velocity $\mathbf{u}_{a}^{\text {ini }}$ and temperature $\theta_{a}^{\text {ini }}$ that is to say

$$
f_{a}^{\mathrm{ini}}(v)=\frac{\rho_{a}^{\mathrm{ini}}}{\left(2 \pi \theta_{a}^{\mathrm{ini}}\right)^{3 / 2}} \exp \left(-\frac{\left|v-u_{a}^{\mathrm{ini}}\right|^{2}}{2 \theta_{a}^{\mathrm{ini}}}\right), \quad a \in\{D, T\} .
$$

We use the physical values given in Table 1 and the values of numerical parameters given in Table 3. Moreover, the initial density functions $f_{\alpha}^{\text {ini }}(v)$ and $f_{n}^{\text {ini }}(v)$ are equal to zero. The initial time step $\Delta t^{0}$ is chosen in such a way condition (33) is satisfied for the proposed test-cases.

\begin{tabular}{ccccc}
\hline $\begin{array}{c}m_{D} \\
(\mathrm{~kg})\end{array}$ & $\begin{array}{c}m_{T} \\
(\mathrm{~kg})\end{array}$ & $\begin{array}{c}m_{\alpha} \\
(\mathrm{kg})\end{array}$ & $\begin{array}{c}m_{n} \\
(\mathrm{~kg})\end{array}$ & $\begin{array}{c}\mathrm{Q} \\
(\mathrm{MeV})\end{array}$ \\
\hline $3.34 \cdot 10^{-27}$ & $5.01 \cdot 10^{-27}$ & $6.68 \cdot 10^{-27}$ & $1.67 \cdot 10^{-27}$ & 17.59 \\
\hline
\end{tabular}

TABLE 1. Physical constants.

\begin{tabular}{cccccc}
\hline$\rho_{D}^{\text {ini }}$ & $\rho_{T}^{\text {ini }}$ & $\begin{array}{c}\theta_{D}^{\text {ini }} \\
(\mathrm{keV})\end{array}$ & $\begin{array}{c}\theta_{T}^{\text {ini }} \\
(\mathrm{keV})\end{array}$ & $\begin{array}{c}\mathbf{u}_{D}^{\text {ini }} \\
\left(\mathrm{m} \cdot \mathrm{s}^{-1}\right)\end{array}$ & $\begin{array}{c}\mathbf{u}_{T}^{\text {ini }} \\
\left(\mathrm{m} \cdot \mathrm{s}^{-1}\right)\end{array}$ \\
\hline $10^{20}$ & $10^{20}$ & 15 & 20 & $\mathbf{0}$ & $\mathbf{0}$ \\
\hline
\end{tabular}

\begin{tabular}{cc}
\hline$N_{D}^{0}$ & $\Delta t^{0}$ \\
& $(\mathrm{~s})$ \\
\hline $10^{4}$ & $10^{-1}$ \\
\hline
\end{tabular}

TABLE 2. Initial values of macroscopic quantities and initial numerical parameters.

\subsection{Validation with a Maxwellian cross-section}

We first validate the numerical method proposed in $\S 2.2$ for a Maxwellian cross-section (9), with $\lambda=10^{-21}$ $\mathrm{m}^{3} \cdot \mathrm{s}^{-1}$, thanks to the explicit time evolution of macroscopic quantities obtained in $\S 1.4$. We can see on Figure 2 that the evolution of the number densities of all species are very close to the one of the explicit solution (24). 


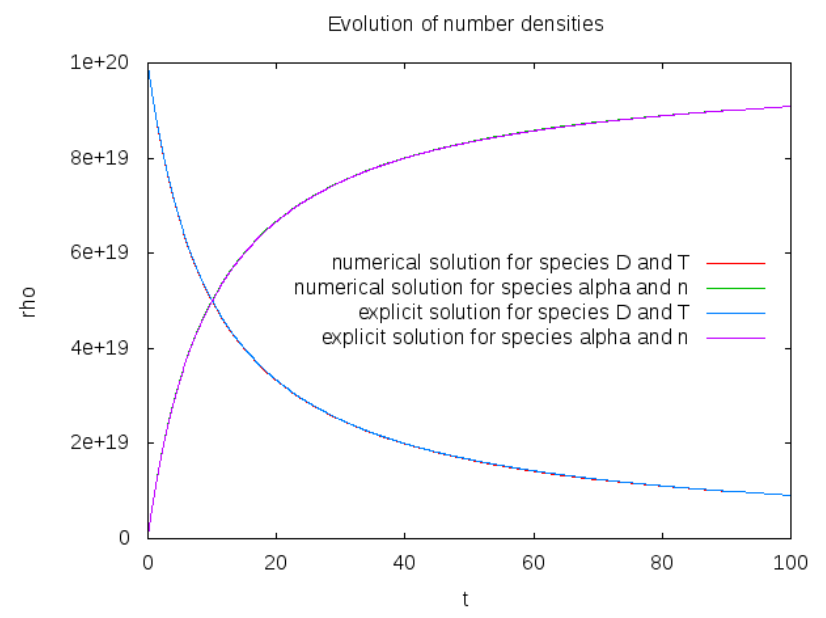

Figure 2. Time evolution of number densities $\rho_{a}, a \in\{D, T, \alpha, n\}$ : comparison between the numerical solutions obtained with Algorithm 2 and explicit solutions given by expressions (24).

Figure 3 presents the density functions $f_{D, 1}^{h}$ and $f_{T, 1}^{h}$ of the first component of the velocity of $D$ and $T$ ions at different times. The density functions are reconstructed from the values of the velocities of numerical particles with a convolution based on a B-spline of order 3 like in [15]:

$$
f_{a, x}^{h, n}\left(t^{n}, v_{x}\right)=\left(S_{3, h} * f_{a, x}^{n}\right)\left(v_{x}\right)=\int_{\mathbb{R}} S_{3, h}\left(v_{x}-v^{\prime}\right) f_{a, x}^{n}\left(v^{\prime}\right) d v^{\prime}=\sum_{i=1}^{N_{a}^{n}} \omega S_{3}\left(v_{x}-V_{i, a, x}^{n}\right) \quad\left(v_{x} \in \mathbb{R}\right),
$$

where

$$
S_{3, h}(y)=\frac{1}{6 h} \begin{cases}\left(2-\frac{|y|}{h}\right)^{3} & \text { if } h \leq|y|<2 h, \\ 4-6\left(\frac{y}{h}\right)^{2}+3\left(\frac{|y|}{h}\right)^{3} & \text { if } 0 \leq|y|<h, \\ 0 & \text { otherwise. }\end{cases}
$$

We can observe that if initially $f_{a, x}^{\mathrm{ini}}(a \in\{D, T\})$ is a Maxwellian distribution, it seems that it remains a Maxwellian distribution at $t>0$ which is in agreement with (20). Moreover, Figure 4 shows that the kinetic temperatures of every species are constant as it is predicted by (26).

\subsection{Simulation with the fusion cross-section}

We consider here the model (1) where the cross-section is a fusion cross-section $\sigma_{f}$ given by (8) with the values of Duane coefficients given by Table 3. Figure 5 presents the time evolution of the number densities of $D$ and $T$ ions on one hand and of $\alpha$ particles and neutrons on the other hand, and the evolution of the total energy of the plasma $E_{\text {tot }}$.

Figure 6 presents the time evolution of kinetic temperatures (in $\mathrm{keV}$ ) of the species. We observe that the kinetic temperatures of $D$ and $T$ are not constant but are decreasing, contrary to the case of the Maxwellian cross-section (compare with Figure 4). Moreover, if we compute the values of the kinetic energy of $\alpha$ particles 

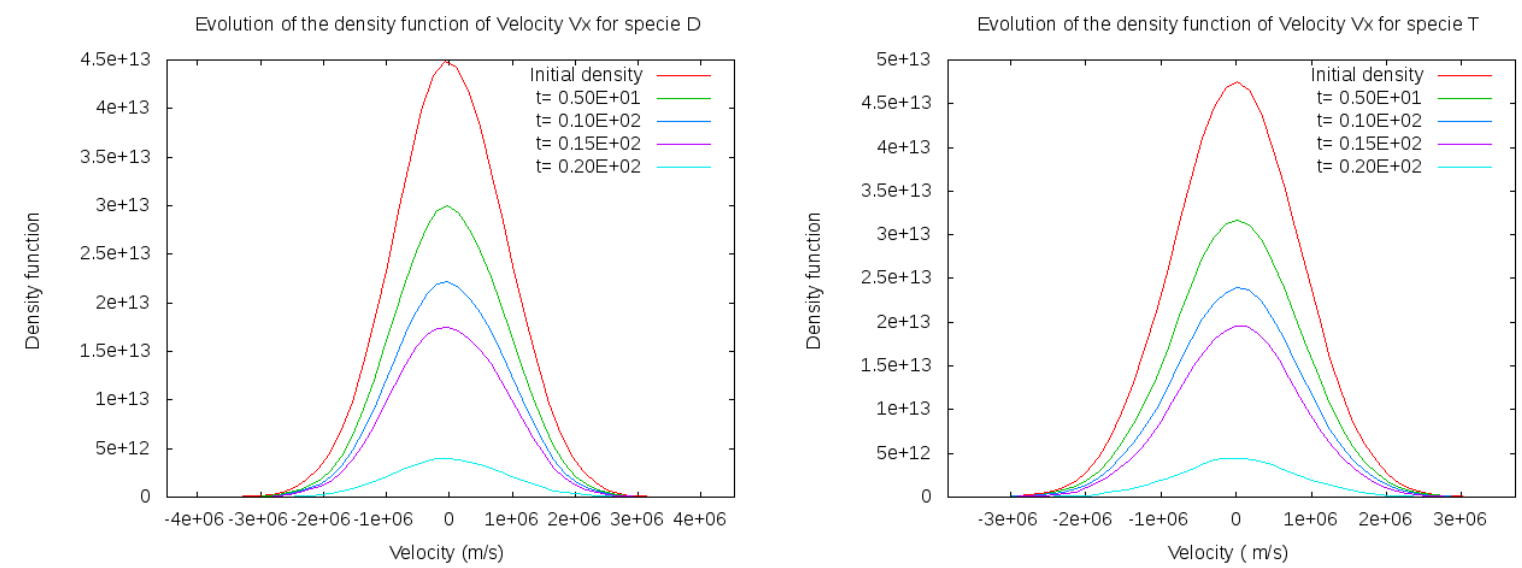

FiguRE 3. Reconstructed density functions of the first component of the velocity of Deuterium and Tritium ions at different instants in the case of the Maxwellian cross-section.
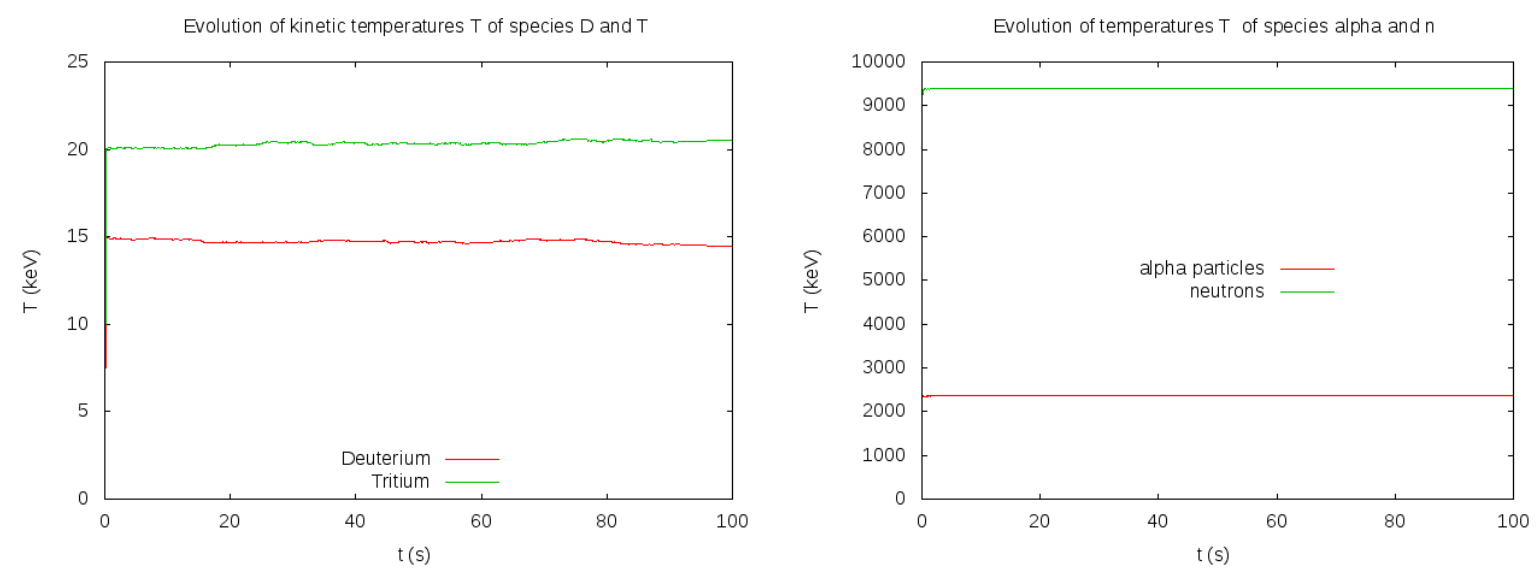

Figure 4. Time evolution of kinetic temperatures in the case of the Maxwellian cross-section.

\begin{tabular}{ccccc}
\hline $\begin{array}{c}p_{1} \\
\left(\mathrm{keV}^{1 / 2}\right)\end{array}$ & $\begin{array}{c}p_{2} \\
\left(\mathrm{~m}^{2}\right)\end{array}$ & $\begin{array}{c}p_{3} \\
\left(\mathrm{keV}^{-1}\right)\end{array}$ & $p_{4}$ & $\begin{array}{c}p_{5} \\
\left(\mathrm{~m}^{2}\right)\end{array}$ \\
\hline 45.95 & $5.02 \cdot 10^{-24}$ & $1.368 \cdot 10^{-2}$ & 1.076 & $4.09 \cdot 10^{-26}$ \\
\hline
\end{tabular}

TABlE 3. Values of Duane coefficients in fusion cross-section $\sigma_{f}(8)$.

and of neutrons from kinetic temperatures $T_{\alpha}$ and $T_{n}$ of Figure 6, we obtain

$$
\frac{3}{2} T_{\alpha} \simeq 3.5 \mathrm{MeV} \quad \text { and } \quad \frac{3}{2} T_{n} \simeq 14.1 \mathrm{MeV},
$$

which are very close to standard values (16) of energy of $\alpha$ particles and neutrons. 

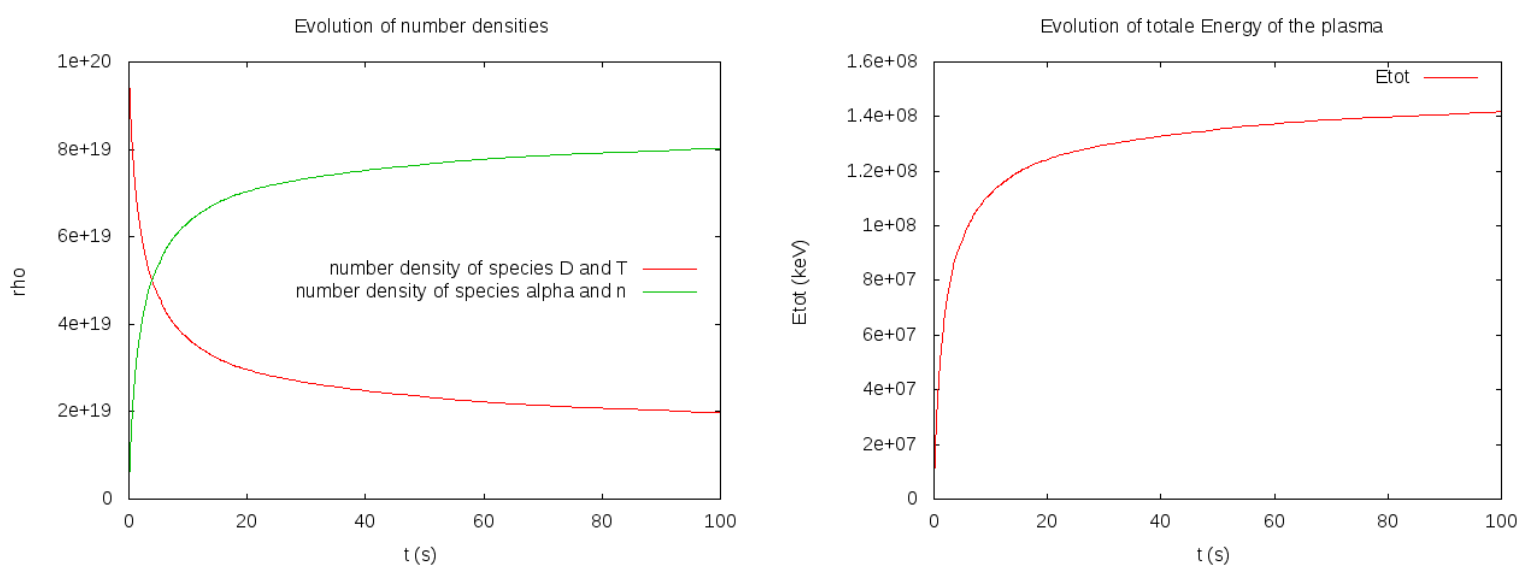

Figure 5. Time evolution of number densities $\rho_{a}, a \in\{D, T, \alpha, n\}$ and of the total energy in the case of the fusion cross-section.
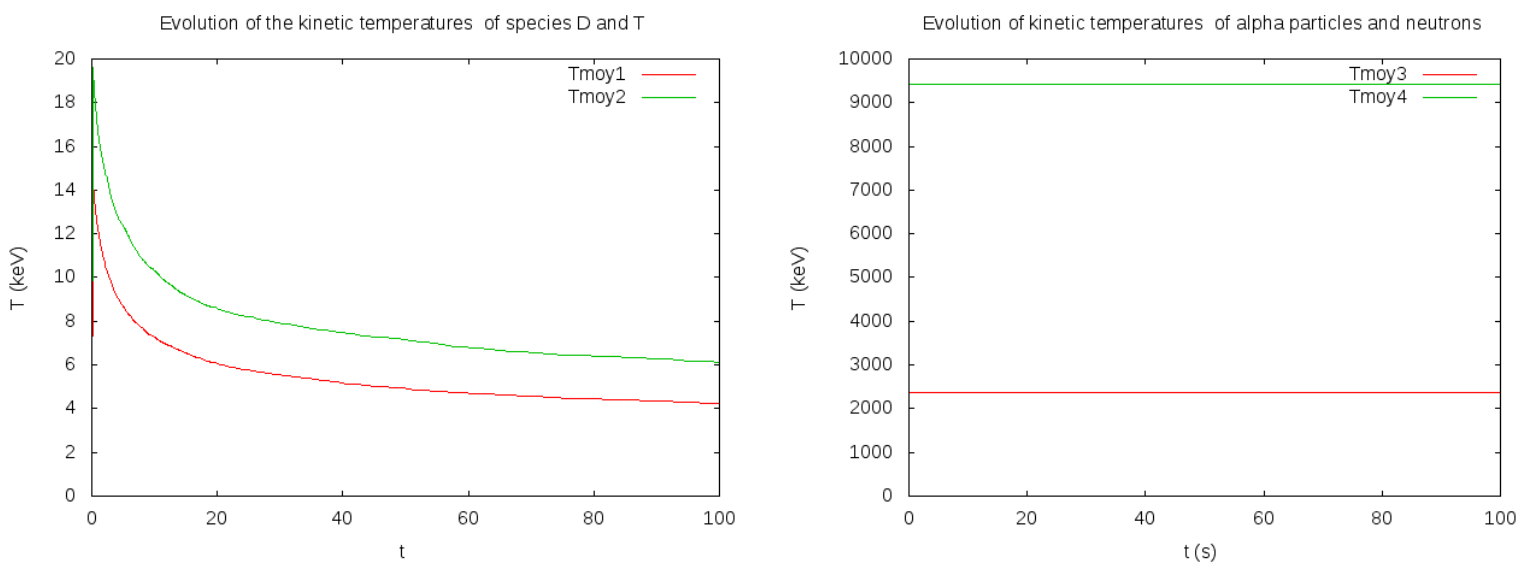

Figure 6. Time evolution of kinetic temperatures in the case of the fusion cross-section.

Figures 7 and 8 present the reconstructed density functions respectively of the first component of the velocity and of the energy of each species. Figure 7 shows that the density functions in velocity of $\alpha$ and $n$ are clearly not Maxwellian distributions. Nevertheless, Figure 8 shows that the energy density functions of $\alpha$ and $n$ seem quite close to a Maxwellian distribution and are centered around the standard values (16).

Finally, we compare on Figure 9 the computed fusion reaction rate $\tau$ with explicit expression (23) which corresponds to a Maxwellian cross-section. Since the parameter $\lambda$ in (23) is a free parameter, we compute it from the value $\tau_{\text {fusion }}^{0}$ of $\tau$ computed at $t=0$ for the fusion cross-section: thus, we set $\lambda=\tau_{\text {fusion }}^{0} /\left(\rho^{\text {ini }}\right)^{2}$. This explicit expression gives a quite good approximation of the computed values of $\tau$ as we see on Figure 9. This expression should be compared to results of computations of the fusion rate obtained in [6] or in [7].

\subsection{On the opportunity to use the asymptotic model}

We now discuss the opportunity to use the asymptotic model (18) instead of model (1). Figure 10 presents the reconstructed energy density function of $\alpha$ particles for different values of $\theta=\min \left(\theta_{D}, \theta_{T}\right)$ at time $t=10^{7}$ 


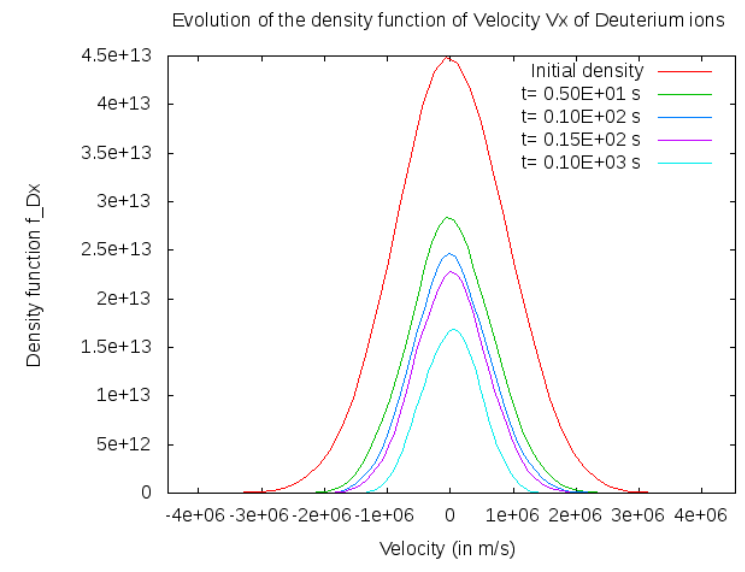

Evolution of the density function of Velocity $V x$ of alpha particles

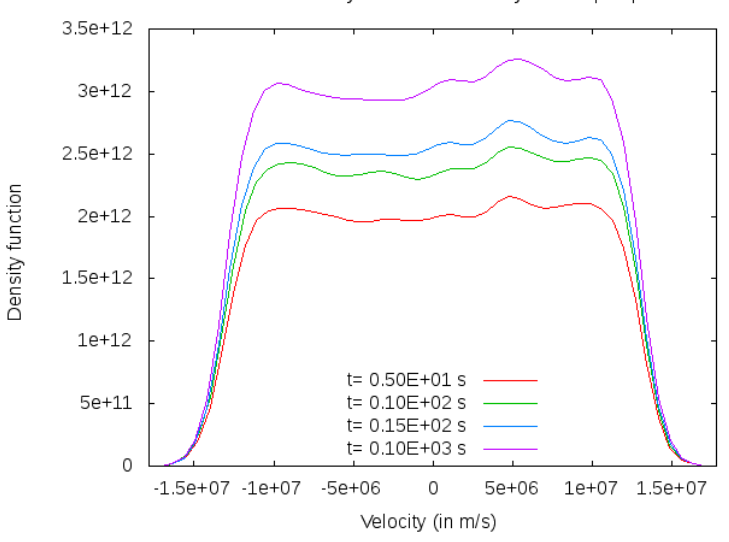

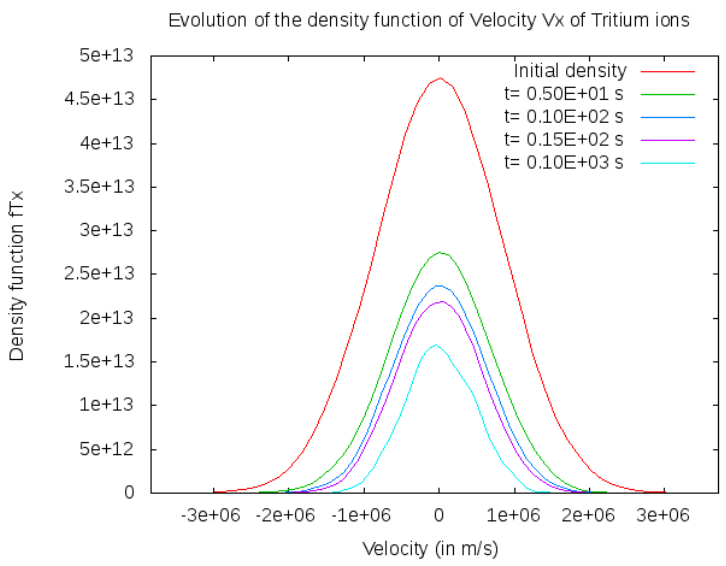

Evolution of the density function of Velocity $V x$ of neutrons

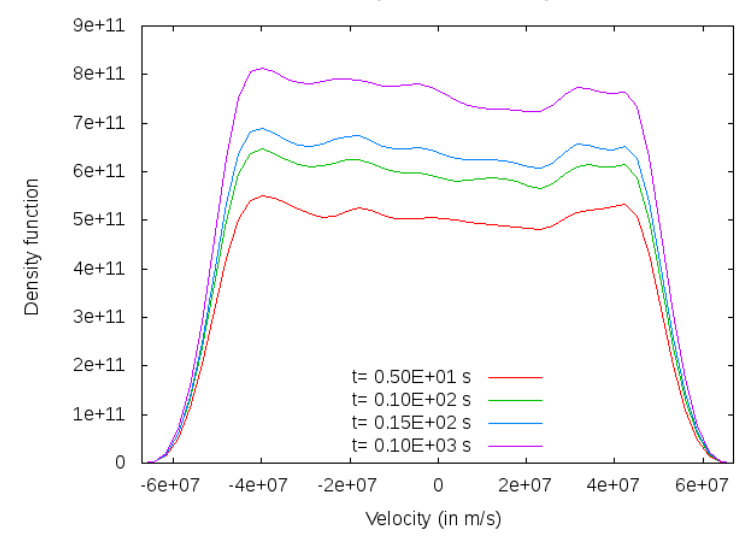

FIgURE 7. Reconstructed density functions of the first component of the velocity for each species in the case of the fusion cross-section.

s (at this time, almost every possible fusion reactions have been made and $f_{\alpha}(t, v)$ is close to $\lim _{t \rightarrow+\infty} f_{\alpha}(t, v)$ ). Initial conditions are again given by (19) with the initial density $f_{D}^{\text {ini }}$ and $f_{T}^{\text {ini }}$ given by (46) with parameters (3) except for the initial kinetic temperatures $\theta_{D}^{\text {ini }}$ and $\theta_{T}^{\text {ini }}$. Indeed, we now take $\theta_{D}^{\text {ini }}=\theta_{T}^{\text {ini }} \in\{5,10,15,20\} \mathrm{keV}$. We see that the sprawl of the distributions in energy of $\alpha$ particles becomes smaller when $\theta$ decreases. This confirms at the discrete level that the energy density functions of $\alpha$ becomes closer to a Dirac mass centered at energy $\overline{E_{\alpha}}$ (given by (16)) when the parameter $\varepsilon$ defined by (15) tends to zero. We verify that this numerical result is also valid for the neutrons (by replacing $\overline{E_{\alpha}}$ with $\overline{E_{n}}$ ). Consequently, when the temperature of the plasma (that is to say $\theta_{D}^{\text {ini }}$ and $\left.\theta_{T}^{\text {ini }}\right)$ is not too high, it seems to be valid to approach model (1) by (18). However, as explained in Remark 6, the numerical cost of the resolution of model (18) is the same as the one of the resolution of model (1) because of the calculus of the fusion reaction rate $\tau(t)$. Then, an interesting possibility to compute evolution of $\alpha$ particles and neutrons could be to use (45) with the explicit expression (23) of $\tau(t)$ since Figure 9 shows that this expression - with an adequate value of $\lambda$ - is a good approximation of the fusion reaction rate. Nevertheless, we should make further investigations to compare the evolution of the distributions in velocity of $\alpha$ particles and neutrons of model (45) with model (1), in particular in the case of non Maxwellian initial Deuterium and Tritium distribution densities. 

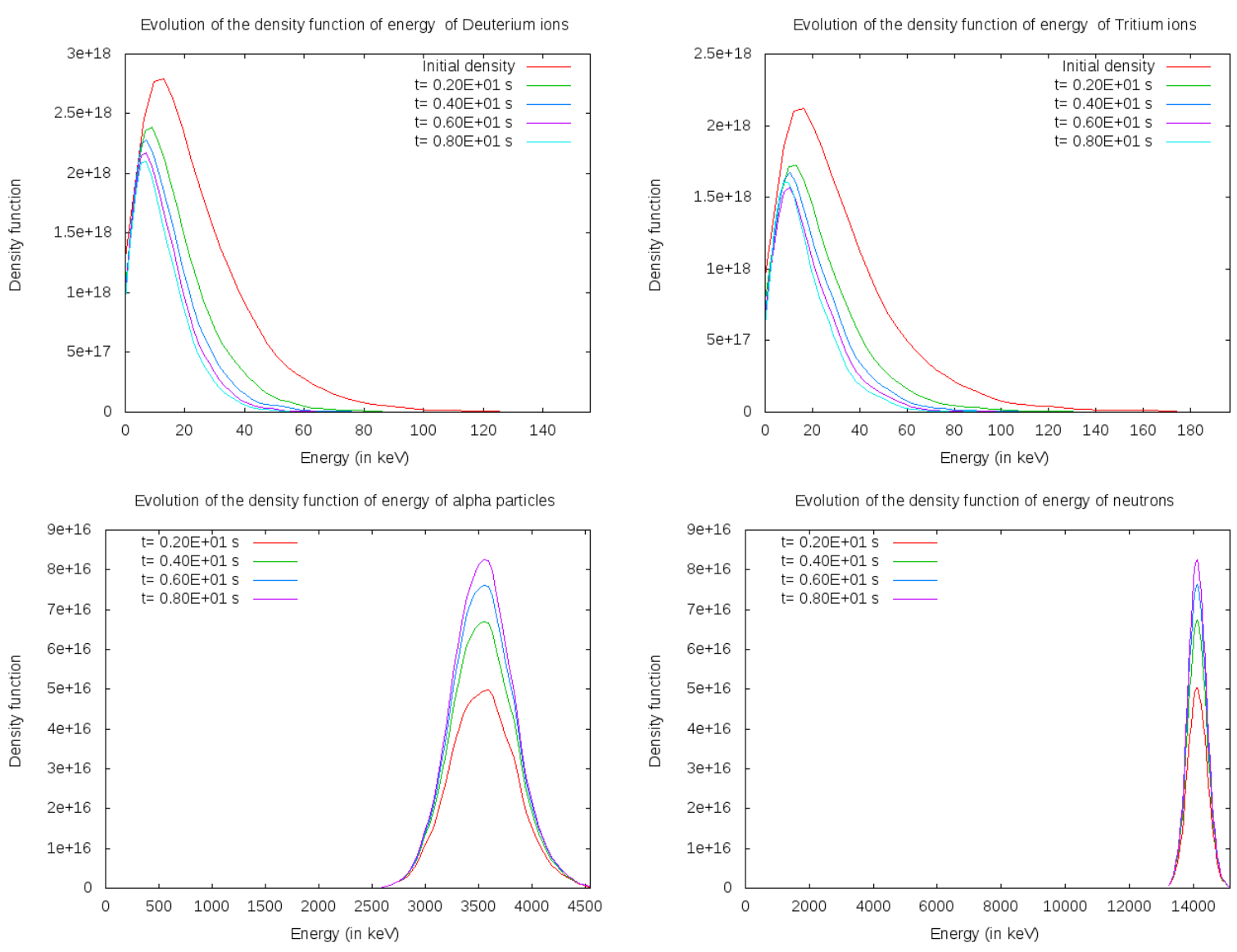

FiguRE 8. Reconstructed energy density functions in the case of the fusion cross-section.

\section{CONCLUSION}

We have proposed and justified a Monte-Carlo algorithm - adapted from a Monte-Carlo algorithm for the Boltzmann equation - to resolve the homogeneous kinetic model (1) describing the nuclear fusion reaction between a Deuterium ion and a Tritium ion and giving an $\alpha$ particle and a neutron. We have also developed a spatially homogeneous code (3D in velocity) based on this Monte-Carlo algorithm. We can thus obtain the evolution of the distributions in velocity, distributions in energy, and macroscopic quantities of Deuterium and Tritium ions, $\alpha$ particles and neutrons starting from initial density functions. The initial distributions used in the presented examples are only Maxwellian distributions but we can also use other initial distributions such as a sum of Maxwellian distributions with different densities, velocities and temperatures.

We have validated the proposed Monte-Carlo algorithm thanks to the use of a Maxwellian cross-section instead of the fusion cross-section. Indeed, the use of a Maxwellian cross-section allows to obtain explicit solutions. Moreover, the numerical cost of our computations is very moderate: a few minutes for a computation with physical and numerical parameters of Table 1 and almost immediate if the number of initial numerical particles $N_{D}^{0}$ is $10^{3}$ instead of $10^{4}$. However, it could be interesting to have a model less costly in order to use it in non-homogeneous simulations with thousands of cells. If we are only interested in the evolution of $\alpha$ particles - because they are the ones who can trigger instabilities in the plasma - and neutrons, a possibility could be to use asymptotic model (45) with an explicit value of the fusion reaction rate $\tau(t)$. This method is already used 


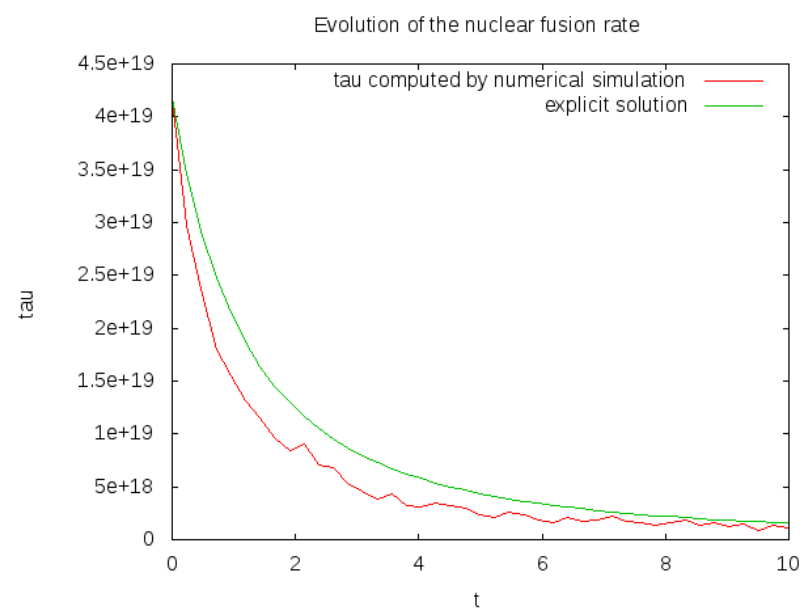

Figure 9. Comparison between the time evolution of the computed fusion rate $\tau$ in the case of fusion cross-section and the explicit expression (23) of $\tau$ in the case of the Maxwellian crosssection.

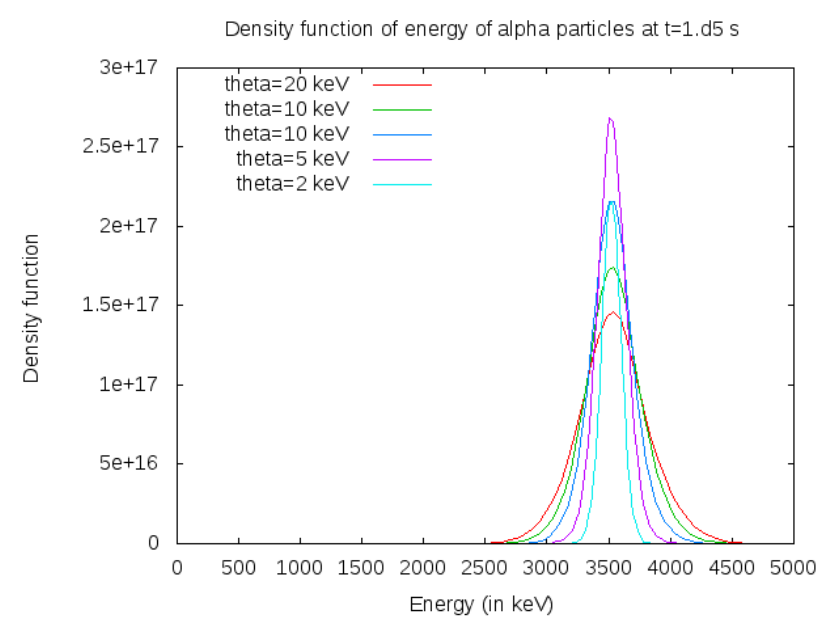

FiguRE 10. Reconstructed energy density function of $\alpha$ particles for different initial values of $\theta=\min \left(\theta_{D}, \theta_{T}\right)$.

as for example in [5]. Here, we proposed to approach the fusion reaction rate $\tau(t)$ by using the explicit formula obtained when the cross-section is a Maxwellian cross-section. Nevertheless, further investigations should be made to validate this approach, especially when the plasma is not initially at the thermodynamical equilibrium.

Finally, we wish to underline that this study is a first step for the study of a more complete model with feedback effect of $\alpha$ particles on Deuterium-Tritium plasma. 


\section{Annex A: proof of Propositions 3 And 4}

Proof. We use the fact that for every integer $N \leq \min \left(N_{D}^{n}, N_{T}^{n}\right)$ and every function $g:\left\{1, \ldots, N_{D}^{n}\right\} \times$ $\left\{1, \ldots, N_{T}^{n}\right\} \mapsto \mathbb{R}$, we have

$$
\frac{1}{N_{D}^{n}} \frac{1}{N_{T}^{n}} \sum_{i=1}^{N_{D}^{n}} \sum_{j=1}^{N_{T}^{n}} g(i, j)=\frac{1}{\sharp P} \frac{1}{N} \sum_{P} \sum_{k=1}^{N} g\left(r_{k}, s_{k}\right),
$$

where $P$ is the set of possible ways to make $N$ distinct pairs $\left(r_{k}, s_{k}\right)_{\{1 \leq k \leq N\}}$ among $\left\{1, \ldots, N_{D}^{n}\right\} \times\left\{1, \ldots, N_{T}^{n}\right\}$. For each test function $\varphi$, we get

$$
\begin{aligned}
\Delta t \int_{\mathbb{R}^{3}} \varphi(v) P_{D}\left(f_{D}^{n}, f_{T}^{n}\right)(v) d v & =\Delta t \lambda_{\max } \int_{\mathbb{R}^{3}} \int_{\mathbb{R}^{3}} \varphi\left(v_{D}\right) f_{D}^{n}\left(v_{D}\right) f_{T}^{n}\left(v_{T}\right) \frac{q\left(\left|v_{D}-v_{T}\right|\right)}{q_{\max }} d v_{D} d v_{T} \\
& =\Delta t \lambda_{\max } \omega^{2} \sum_{i=1}^{N_{D}^{n}} \sum_{j=1}^{N_{T}^{n}} \varphi\left(V_{D, i}^{n}\right) p_{i, j}^{n} \\
& =\frac{\omega \eta^{n}}{\sharp P} \sum_{P} \sum_{k=1}^{\mathcal{N}^{n}} \varphi\left(V_{D, r_{k}}^{n}\right) \int_{0}^{1} \mathbb{1}_{\left\{0 \leq u \leq p_{r_{k}, s_{k}}^{n}\right\}} d u,
\end{aligned}
$$

where $\mathcal{N}^{n}$ is defined by (35) and where we have set

$$
p_{i, j}^{n}=\frac{q\left(\left|V_{D, i}^{n}-V_{T, j}^{n}\right|\right)}{q_{\max }} .
$$

For the gain term, we have:

$$
\begin{aligned}
\Delta t \int_{\mathbb{R}^{3}} \varphi(v) G_{\alpha}\left(f_{D}^{n}, f_{T}^{n}\right)(v) d v & =\lambda_{\max } \Delta t \int_{\mathbb{S}^{2}} \int_{\mathbb{R}^{3}} \int_{\mathbb{R}^{3}} \varphi\left(v_{\alpha}\right) f_{D}^{n}\left(v_{D}\right) f_{T}^{n}\left(v_{T}\right) \frac{q\left(\left|v_{D}-v_{T}\right|\right)}{q_{\max }} d v_{D} d v_{T} \frac{d \Omega}{4 \pi} \\
& =\lambda_{\max } \Delta t \omega^{2} \sum_{i=1}^{N_{D}^{n}} \sum_{j=1}^{N_{T}^{n}} \int_{\mathbb{S}^{2}} \varphi\left(V_{\alpha, i, j, \Omega}^{n}\right) p_{i, j} \frac{d \Omega}{4 \pi} \\
& =\frac{\omega \eta^{n}}{\sharp P} \sum_{P} \sum_{k=1}^{\mathcal{N}^{n}} \int_{\mathbb{S}^{2}} \int_{0}^{1} \varphi\left(V_{\alpha, r_{k}, s_{k}, \Omega}^{n}\right) \mathbb{1}_{\left\{0 \leq u \leq p_{r_{k}, s_{k}}^{n}\right\}} d u \frac{d \Omega}{4 \pi} .
\end{aligned}
$$

\section{REFERENCES}

[1] Bird, G.A. Molecular gas dynamics and the direct simulation of gas flows. Oxford University Press (1994).

[2] Charles, F. Modélisation mathématique et étude numérique d'un aérosol dans un gaz raréfié. Application à la simulation du transport de particules de poussière en cas d'accident de pert de vide dans ITER. PhD Thesis, École Normale Supérieure de Cachan, p. 69-115 (2009).

[3] Dellacherie, S. and Sentis, R. Nuclear Collisions Models with Boltzmann Operators. Mathematical Models and Methods in Applied Sciences, 10, No 4, p. 479-506 (2000).

[4] Duane, B.H. Fusion cross-section theory. Rapport du Battelle Pacific Northwest Laboratory (Université du Michigan), BNWL1685 (1951).

[5] Farengo, R. and Zarco, M. and Ferrari, H.E. Simulation of alpha particle current drive and heating in spherical tokamaks. Proceeding of 12th IAEA Technical Meeting on Energetic Particles in Magnetic Confinement Systems (2011).

[6] Goncharov, P.R. Practical Calculation of Nuclear Fusion Power for a Toroidal Plasma Device with Magnetic Confinement. Plasma and Fusion Research, J-STAGE, 5, No 0, p. 1028-1028 (2010).

[7] Heltemes, T.A. and Moses, G.A. and Santarius, J.F. Analysis of an Improved Fusion Reaction Rate Model for Use in Fusion Plasma Simulations. Technická zpráva, Duben (2005). 
[8] Huba, J.D. NRL plasma formulary. Naval Research Laboratory, p. 44-45 (1980).

[9] Lapeyre, B. and Pardoux, E. and Sentis, R. Méthodes de Monte-Carlo pour les équations de transport et de diffusion. Springer (1998).

[10] Larroche, O. Kinetic simulation of a plasma collision experiment. Phys. Fluids B5, p. 2816-2840 (1993).

[11] Li, X.Z. and Liu, B. and Chen, S. and Wei, Q.M. and Hora, H. Fusion cross-sections for inertial fusion energy. Laser and Particle Beams, Cambridge Univ Press 22, No 4, p. 469-477 (2004).

[12] Pareschi, L. and Russo, G. An introduction to Monte Carlo methods for the Boltzmann equation. In ESAIM: Proceedings (1999).

[13] Perthame, B. Introduction to the theory of random particle methods for Boltzmann equation. Technical Report 2218, INRIA (1994).

[14] Villani, C. A review of mathematical topics in collisional kinetic theory. Handbook of mathematical fluid dynamics, 1, p. $71-305$ (2002).

[15] Wollman, S. On the approximation of the Vlasov-Poisson system by particle methods. SIAM J. Numer. Anal., 37 p. $1369-1398$ (2000). 